\title{
Application of Lie group analysis to a core group model for sexually transmitted diseases
}

\author{
$M E D W A R D S^{a}$ and $M C N U C C I^{b}$ \\ a School of Mathematics and Applied Statistics, University of Wollongong, Wollongong \\ NSW 2522, Australia, \\ E-mail: maureen_edwards@uow.edu.au \\ ${ }^{b}$ Dipartimento di Matematica e Informatica, Università di Perugia, 06123 Perugia, Italy, \\ E-mail:nucci@unipg.it
}

Received May 13, 2005; Accepted in Revised Form November 23, 2005

\begin{abstract}
Lie group analysis is applied to a core group model for sexually transmitted disease formulated by Hadeler and Castillo-Chavez [Hadeler K P and Castillo-Chavez C, A core group model for disease transmission, Math. Biosci. 128 (1995), 41-55]. Several instances of integrability even linearity are found which lead to the general solution of the model. A discussion of such solutions is presented and it is shown how they complement Hadeler and Castillo-Chavez's qualitative analysis.
\end{abstract}

\section{Introduction}

In [8] Hadeler and Castillo-Chavez presented a model for sexually transmitted diseases which takes into consideration an active and relatively small core group of constant size. The core group recruits individuals from the non-core group, and the rate of recruitment may depend on the state of the core group. The non-core group is completely inactive. The total population has size $P(t)$, and the non-core group has size $A$. The population of the core group $C$ is further divided into susceptibles $S$, educated (or vaccinated) $V$, and infecteds $I$. The birth rate is $b>0$, the birth rate of infecteds is $\tilde{b} \leq b, \tilde{b} \geq 0$, the death rate is $\mu>0$, the death rate of infecteds is $\tilde{\mu} \geq \mu$, the recovery rate is $\alpha \geq 0$, the education (vaccination) rate is $\psi \geq 0$, the transmission rate from infecteds to susceptibles is $\beta \geq 0$, the transmission rate from infected to educated (vaccinated) is $\tilde{\beta}, 0 \leq \tilde{\beta} \leq \beta$. At recovery individuals may either pass into the educated class at the rate $\alpha \gamma, 0 \leq \gamma \leq 1$, or return to the susceptible class at the rate $\alpha(1-\gamma)$. Recruitment into the core group is described by a function $r(I, C)$. Hadeler and Castillo-Chavez focused on the situation where the disease has no demographic effects and the population size is constant, i.e. $P=$ const, $b=\tilde{b}=\mu=\tilde{\mu}$. Thus their model assumes the form

$$
\begin{aligned}
\dot{A} & =\mu P-A r(I, C)-\mu A, \\
\dot{S} & =\operatorname{Ar}(I, C)-\beta \frac{S I}{C}-\psi S+\alpha(1-\gamma) I-\mu S,
\end{aligned}
$$




$$
\begin{aligned}
\dot{V} & =\psi S-\tilde{\beta} \frac{V I}{C}+\alpha \gamma I-\mu V, \\
\dot{I} & =\frac{\beta S I+\tilde{\beta} V I}{C}-\alpha I-\mu I .
\end{aligned}
$$

They point out that this system is closely related to a model for an isolated core population of constant size $C=1$, i.e.

$$
\begin{aligned}
\dot{S} & =\mu-\beta S I-\psi S+\alpha(1-\gamma) I-\mu S \\
\dot{V} & =\psi S-\tilde{\beta} V I+\alpha \gamma I-\mu V \\
\dot{I} & =\beta S I+\tilde{\beta} V I-\alpha I-\mu I .
\end{aligned}
$$

In [8] the stationary solutions of system (1.5)-(1.7) are found and their qualitative features discussed. Then the stationary solutions of system (1.1)-(1.4) are also discussed. Qualitative conclusions are finally drawn.

In the present paper we apply Lie group analysis to system (1.5)-(1.7) in order to determine under which physical conditions on the parameters Lie point symmetries exist and, when possible, deduce the general solution in closed form. We also discuss the solutions that we have found and show how our work complements Hadeler and Castillo-Chavez's qualitative analysis.

\section{Lie group analysis}

In January 2001 the first Whiteman prize for notable exposition on the history of mathematics was awarded to Thomas Hawkins by the American Mathematical Society. In the citation, published in the Notices of AMS 48 416-417 (2001), one reads that Thomas Hawkins "... has written extensively on the history of Lie groups. In particular he has traced their origins to [Lie's] work in the 1870s on differential equations ... the idée fixe guiding Lie's work was the development of a Galois theory of differential equations". Also Hawkins had established "the nature and extent of Jacobi's influence upon Lie" [9]. This is particularly noteworthy since 2004 marked two hundred years since Jacobi's birth. "Given the fact that the Jacobi Identity is fundamental to the theory of Lie groups, Jacobi's influence upon Lie will come as no surprise. But the bald fact that he inherited the Identity from Jacobi fails to convey fully or accurately the historical dimension of the impact of Jacobi's work on partial differential equations" [9].

Lie's monumental work on transformation groups, [18], [19] and [20], and in particular contact transformations [21], has provided systematic techniques for obtaining exact solutions of differential equations [22].

Many books have been dedicated to this subject and its generalizations ([1], [3], [33], [32], [4], [35], [36], [11], [13], [14], [15], [12], [2]).

Lie group analysis is indeed the most powerful tool to find the general solution of ordinary differential equations. Any known integration technique can be shown to be a particular case of a general integration method based on the derivation of the continuous group of symmetries admitted by the differential equation, i.e. the Lie symmetry algebra. The admitted Lie symmetry algebra can be derived easily by a straightforward although lengthy procedure. As computer algebra software becomes widely used, the integration of systems of ordinary differential equations by means of Lie group analysis is becoming easier to 
perform. A major drawback of Lie's method is that it is useless when applied to systems of $n$ first-order equations, because they admit an infinite number of symmetries, and there is no systematic way to find even an one-dimensional Lie symmetry algebra, apart from trivial groups like translations in time admitted by autonomous systems. One may try to derive an admitted $n$-dimensional solvable Lie symmetry algebra by making an ansatz on the form of its generators but when successful (rarely) it is just a lucky guess.

However, in [27] Nucci has remarked that any system of $n$ first-order equations could be transformed into an equivalent system where at least one of the equations is of second order. Then the admitted Lie symmetry algebra is no longer infinite dimensional, and nontrivial symmetries of the original system could be retrieved [27]. This idea has been successfully applied in several instances ([27], [37], [30], [23], [28], [31], [17], [29]). Here we apply this method to system (1.5)-(1.7) and employ ad hoc interactive programs [25], [26] written in REDUCE language to calculate the admitted Lie algebra.

System (1.5)-(1.7) is composed of three first-order ordinary differential equations which can be easily reduced to two equations by using the condition

$$
C \equiv 1=S+V+I
$$

Then we can easily transform the system of two equations so obtained into one equation of second order. We derive $I$ from (2.1), i.e.

$$
I=1-S-V,
$$

and then deduce $V$ from equation (1.5), i.e.

$$
V=\frac{\dot{S}-\mu S+\psi S-\mu}{\alpha \gamma-\alpha+\beta S}-S+1
$$

Then a second order equation for $S$ is obtained, i.e

$$
\ddot{S}=f(t, S, \dot{S}),
$$

and we search for its Lie symmetry algebra. An operator $\Gamma$

$$
\Gamma=v(t, u) \partial_{t}+g(t, u) \partial_{u}
$$

is said to generate a Lie point symmetry group of an equation of second-order, say

$$
\ddot{u}=F(t, u, \dot{u}),
$$

if its second prolongation

$$
\Gamma_{2}=\Gamma+\left(\frac{\mathrm{d} g}{\mathrm{~d} t}-\dot{u} \frac{\mathrm{d} v}{\mathrm{~d} t}\right) \partial_{\dot{u}}+\left[\frac{\mathrm{d}}{\mathrm{d} t}\left(\frac{\mathrm{d} g}{\mathrm{~d} t}-\dot{u} \frac{\mathrm{d} v}{\mathrm{~d} t}\right)-\ddot{u} \frac{\mathrm{d} v}{\mathrm{~d} t}\right] \partial_{\ddot{u}}
$$

applied to equation (2.6), on its solutions, is identically equal to zero, i.e.

$$
\left.\Gamma_{2}(2.6)\right|_{(2.6)}=0 .
$$

The determining equation (2.7) constitutes an overdetermined system of linear partial differential equations in the unknowns $v(t, u), g(t, u)$. When we apply Lie group analysis 
to equation (2.4) then we obtain a first-order linear partial differential equation for $v(t, S)$; its characteristic curves suggests making the following simplifying transformation

$$
S=\frac{-\alpha \gamma+\alpha+u}{\beta},
$$

where $u(t)$ is the new dependent variable. Then (2.3) transforms into

$$
V=1+\frac{\dot{u}+\alpha(1-\gamma)(\mu+\psi)+(\alpha \gamma-\alpha+\mu+\psi-u) u-\beta \mu}{\beta u},
$$

and (2.4) becomes

$$
\begin{aligned}
\ddot{u}= & \alpha^{2} \beta \gamma^{2} \mu u+\alpha^{2} \beta \gamma^{2} \psi u-\alpha^{2} \beta \gamma \mu u-\alpha^{2} \beta \gamma \psi u+\alpha^{2} \tilde{\beta} \gamma^{2} \mu^{2}+2 \alpha^{2} \tilde{\beta} \gamma^{2} \mu \psi \\
& -\alpha^{2} \tilde{\beta} \gamma^{2} \mu u+\alpha^{2} \tilde{\beta} \gamma^{2} \psi^{2}-\alpha^{2} \tilde{\beta} \gamma^{2} \psi u-2 \alpha^{2} \tilde{\beta} \gamma \mu^{2}-4 \alpha^{2} \tilde{\beta} \gamma \mu \psi+2 \alpha^{2} \tilde{\beta} \gamma \mu u \\
& -2 \alpha^{2} \tilde{\beta} \gamma \psi^{2}+2 \alpha^{2} \tilde{\beta} \gamma \psi+\alpha^{2} \tilde{\beta} \mu^{2}+2 \alpha^{2} \tilde{\beta} \mu \psi-\alpha^{2} \tilde{\beta} \mu u+\alpha^{2} \tilde{\beta} \psi^{2} \\
& -\alpha^{2} \tilde{\beta} \psi u+\alpha \beta^{2} \gamma \mu u+2 \alpha \beta \tilde{\beta} \gamma \mu^{2}+2 \alpha \beta \tilde{\beta} \gamma \mu \psi-2 \alpha \beta \tilde{\beta} \gamma \mu u-\alpha \beta \tilde{\beta} \gamma \psi u \\
& -2 \alpha \beta \tilde{\beta} \mu^{2}-2 \alpha \beta \tilde{\beta} \mu \psi+2 \alpha \beta \tilde{\beta} \mu u+\alpha \beta \tilde{\beta} \psi u+\alpha \beta \gamma \mu^{2} u \\
& +\alpha \beta \gamma \mu \psi u-2 \alpha \beta \gamma \mu u^{2}-\alpha \beta \gamma \mu \dot{u}-2 \alpha \beta \gamma \psi u^{2}-\alpha \beta \gamma \psi \dot{u}-\alpha \beta \gamma u \dot{u}-\alpha \beta \mu^{2} u \\
& -\alpha \beta \mu \psi u+\alpha \beta \mu u^{2}+\alpha \beta \mu \dot{u}+\alpha \beta \psi u^{2}+\alpha \beta \psi \dot{u}-2 \alpha \tilde{\beta} \gamma \mu^{2} u-4 \alpha \tilde{\beta} \gamma \mu \psi u \\
& +2 \alpha \tilde{\beta} \gamma \mu u^{2}-2 \alpha \tilde{\beta} \gamma \mu \dot{u}-2 \alpha \tilde{\beta} \gamma \psi^{2} u+2 \alpha \tilde{\beta} \gamma \psi u^{2}-2 \alpha \tilde{\beta} \gamma \psi \dot{u}+\alpha \tilde{\beta} \gamma u \dot{u} \\
& +2 \alpha \tilde{\beta} \mu^{2} u+4 \alpha \tilde{\beta} \mu \psi u-2 \alpha \tilde{\beta} \mu u^{2}+2 \alpha \tilde{\beta} \mu \dot{u}+2 \alpha \tilde{\beta} \psi^{2} u \\
& -2 \alpha \tilde{\beta} \psi u^{2}+2 \alpha \tilde{\beta} \psi \dot{u}-\alpha \tilde{\beta} u \dot{u}+\beta^{2} \tilde{\beta} \mu^{2} \\
& -\beta^{2} \tilde{\beta} \mu u+\beta^{2} \mu^{2} u-\beta^{2} \mu u^{2}-\beta^{2} \mu \dot{u}-2 \beta \tilde{\beta} \mu^{2} u-2 \beta \tilde{\beta} \mu \psi u \\
& +2 \beta \tilde{\beta} \mu u^{2}-2 \beta \tilde{\beta} \mu \dot{u}+\beta \tilde{\beta} \psi u^{2}+\beta \tilde{\beta} u \dot{u}-\beta \mu^{2} u^{2}-\beta \mu \psi u^{2}+\beta \mu u^{3}-\beta \mu u \dot{u} \\
& +\beta \psi u^{3}+\beta u^{2} \dot{u}+\beta \dot{u}^{2}+\tilde{\beta} \mu^{2} u^{2}+2 \tilde{\beta} \mu \psi u^{2}-\tilde{\beta} \mu u^{3}+2 \tilde{\beta} \mu u \dot{u} \\
& \left.+\tilde{\beta} \psi^{2} u^{2}-\tilde{\beta} \psi u^{3}+2 \tilde{\beta} \psi u \dot{u}-\tilde{\beta} u^{2} \dot{u}+\tilde{\beta} \dot{u}^{2}\right) /(\beta u) .
\end{aligned}
$$

We apply Lie group analysis to (2.10) and obtain non-trivial Lie point symmetries in five cases:

\section{Case (1)}

$$
\beta=\tilde{\beta}
$$

In this case we obtain an eight-dimensional Lie symmetry algebra ${ }^{1}$ which is isomorphic to $\operatorname{sl}(3, \mathbb{R})[22],[7]$. This means that equation (2.10) is linearizable [22] by means of a point transformation. In order to find the linearizing transformation we have to look for a two-dimensional abelian intransitive subalgebra and, following Lie's classification of two-dimensional algebras in the real plane [22], we have to transform it into the canonical form

$$
\partial_{\tilde{u}}, \quad \tilde{t} \partial_{\tilde{u}}
$$

\footnotetext{
${ }^{1}$ We do not list the eight operators because of their length. However we will provide them in MAPLE format to anyone interested.
} 
with $\tilde{u}$ and $\tilde{t}$ the new dependent and independent variables, respectively. We found that one such subalgebra is that generated by $\Gamma_{3}$ and $\Gamma_{6}$, i.e.

$$
\begin{aligned}
\Gamma_{3}= & \frac{\tilde{\beta} \mu-\tilde{\beta} u+\alpha u-\alpha \mu-u \psi-\alpha \psi+\alpha \gamma \psi+\alpha \gamma \mu}{u(\mu+\psi)(\tilde{\beta}+\psi-\alpha)(\alpha-\tilde{\beta}+\mu) e^{(\alpha-\tilde{\beta}+\mu) t}}\left[\partial_{t}+(\tilde{\beta} \mu-\tilde{\beta} u+\alpha u\right. \\
& \left.-\alpha \mu-u \psi-\alpha \psi+\alpha \gamma \psi+\alpha \gamma \mu) \partial_{u}\right] \\
\Gamma_{6}= & \frac{1}{u(\mu+\psi)(-\alpha+\tilde{\beta}-\mu) e^{(\mu+\psi) t}}\left[\partial_{t}+(\tilde{\beta} \mu-\tilde{\beta} u+\alpha u-\alpha \mu-u \psi-\alpha \psi\right. \\
& \left.+\alpha \gamma \psi+\alpha \gamma \mu) \partial_{u}\right] .
\end{aligned}
$$

In order to find $\tilde{t}$ and $\tilde{u}$ we have to solve the following four linear partial differential equations of first-order:

$$
\Gamma_{3}(\tilde{t})=0, \quad \Gamma_{6}(\tilde{t})=0, \quad \Gamma_{3}(\tilde{u})=\tilde{t}, \quad \Gamma_{6}(\tilde{u})=1 .
$$

After a lengthy calculation we derive that if $\tilde{\beta} \neq \alpha-\psi$ then

$$
\begin{aligned}
\tilde{t} & =\frac{e^{(\tilde{\beta}-\alpha+\psi) t}}{\alpha-\tilde{\beta}-\psi}(\tilde{\beta} \mu-\tilde{\beta} u+\alpha u-\alpha \mu-u \psi-\alpha \psi+\alpha \gamma \psi+\alpha \gamma \mu), \\
\tilde{u} & =e^{(\mu+\psi) t}(\alpha \gamma \mu+\alpha \gamma \psi-\alpha \mu-\alpha \psi+\tilde{\beta} \mu-\mu u-\psi u)
\end{aligned}
$$

and equation (2.10) becomes

$$
\frac{\mathrm{d}^{2} \tilde{u}}{\mathrm{~d} \tilde{t}^{2}}=0
$$

which can be easily integrated, i.e.

$$
\tilde{u}=a_{1} \tilde{t}+a_{2} .
$$

with $a_{1}, a_{2}$ arbitrary constants. Taking into consideration (2.15), (2.16), (2.8), (2.3) and (2.2) it is easy to obtain the general solution of system (1.5)-(1.7), i.e.

$$
\begin{aligned}
S & =\frac{e^{\tilde{\beta} t}(\alpha(\gamma-1)(\alpha-\tilde{\beta}+\mu)+\tilde{\beta} \mu) a_{1}}{\tilde{\beta}(\tilde{\beta}+\psi-\alpha)\left(e^{(\alpha+\mu) t}(\mu+\psi)+e^{\tilde{\beta} t} a_{1}\right)}+\frac{e^{(\alpha-\psi) t}\left(e^{(\mu+\psi) t} \tilde{\beta} \mu-a_{2}\right)}{\tilde{\beta}\left(e^{(\alpha+\mu) t}(\mu+\psi)+e^{\tilde{\beta} t} a_{1}\right)},(2) \\
V & =\frac{e^{\alpha t}\left(e^{\mu t} \tilde{\beta} \psi+e^{-\psi t} a_{2}\right)}{\tilde{\beta}\left(e^{(\alpha+\mu) t}(\mu+\psi)+e^{\tilde{\beta} t} a_{1}\right)}+\frac{e^{\tilde{\beta} t}(\gamma(\tilde{\beta}-\alpha-\mu)+\psi+\mu) \alpha a_{1}}{\tilde{\beta}(\tilde{\beta}+\psi-\alpha)\left(e^{(\alpha+\mu) t}(\mu+\psi)+e^{\tilde{\beta} t} a_{1}\right)}, \\
I & =\frac{e^{\tilde{\beta} t}(\tilde{\beta}-\mu-\alpha) a_{1}}{\tilde{\beta}\left(e^{(\alpha+\mu) t}(\mu+\psi)+e^{\tilde{\beta} t} a_{1}\right)} .
\end{aligned}
$$

If $\tilde{\beta}=\alpha-\psi$ and $\gamma \neq \frac{\psi(\alpha+\mu)}{\alpha(\mu+\psi)}$, then we obtain that the two-dimensional abelian intransitive subalgebra of the admitted eight-dimensional Lie point symmetry algebra of equation (2.10) generated by the operators:

$$
\begin{aligned}
& X_{6}=\frac{e^{-(\mu+\psi) t}}{u}\left(\partial_{t}-(\alpha \psi+\mu \psi-\alpha \gamma \mu-\alpha \gamma \psi) \partial_{u}\right) \\
& X_{7}=\frac{u+t(\alpha \psi+\mu \psi-\alpha \gamma \mu-\alpha \gamma \psi)}{e^{(\mu+\psi) t} u(\alpha \psi+\mu \psi-\alpha \gamma \mu-\alpha \gamma \psi)}\left(\partial_{t}-(\alpha \psi+\mu \psi-\alpha \gamma \mu-\alpha \gamma \psi) \partial_{u}\right)
\end{aligned}
$$


can be put into the canonical form (2.11) if

$$
\begin{aligned}
\tilde{t}= & \frac{\alpha \psi+\mu \psi-\alpha \gamma \mu-\alpha \gamma \psi}{u+t(\alpha \psi+\mu \psi-\alpha \gamma \mu-\alpha \gamma \psi)} \\
\tilde{u}= & (u+t(\alpha \psi+\mu \psi-\alpha \gamma \mu-\alpha \gamma \psi))^{2} \\
& +\frac{(\alpha \psi+\mu \psi-\alpha \gamma \mu-\alpha \gamma \psi+(\mu+\psi) u)(\alpha \psi+\mu \psi-\alpha \gamma \mu-\alpha \gamma \psi)}{e^{-(\mu+\psi) t}(u+t(\alpha \psi+\mu \psi-\alpha \gamma \mu-\alpha \gamma \psi))(\mu+\psi)^{2}} .
\end{aligned}
$$

Thus equation (2.10) transforms into:

$$
\frac{\mathrm{d}^{2} \tilde{u}}{\mathrm{~d} \tilde{t}^{2}}=-6 \frac{(\alpha \psi+\mu \psi-\alpha \gamma \mu-\alpha \gamma \psi)^{2}}{\tilde{t}^{4}},
$$

which can be easily integrated, i.e.

$$
\tilde{u}=a_{1}+a_{2} \tilde{t}-\frac{(\alpha \psi+\mu \psi-\alpha \gamma \mu-\alpha \gamma \psi)^{2}}{\tilde{t}^{2}} .
$$

Consequently the general solution of (1.5)-(1.7) is:

$$
\begin{aligned}
S= & (\mu+\psi) \frac{\left(a_{1} t+a_{2}\right)(\alpha \psi+\mu \psi-\alpha \gamma \mu-\alpha \gamma \psi)-\alpha a_{1}(1-\gamma)}{\left(e^{(\mu+\psi) t}(\alpha \psi+\mu \psi-\alpha \gamma \mu-\alpha \gamma \psi)-(\mu+\psi) a_{1}\right)(\alpha-\psi)} \\
& +\frac{e^{(\mu+\psi) t}(\alpha \psi+\mu \psi-\alpha \gamma \mu-\alpha \gamma \psi) \mu}{\left(e^{(\mu+\psi) t}(\alpha \psi+\mu \psi-\alpha \gamma \mu-\alpha \gamma \psi)-(\mu+\psi) a_{1}\right)(\mu+\psi)}, \\
V= & -(\mu+\psi) \frac{\left(a_{1} t+a_{2}\right)(\alpha \psi+\mu \psi-\alpha \gamma \mu-\alpha \gamma \psi)+a_{1}(\alpha \gamma+\mu)}{\left(e^{(\mu+\psi) t}(\alpha \psi+\mu \psi-\alpha \gamma \mu-\alpha \gamma \psi)-(\mu+\psi) a_{1}\right)(\alpha-\psi)} \\
& +\frac{e^{(\mu+\psi) t}(\alpha \psi+\mu \psi-\alpha \gamma \mu-\alpha \gamma \psi) \psi}{\left(e^{(\mu+\psi) t}(\alpha \psi+\mu \psi-\alpha \gamma \mu-\alpha \gamma \psi)-(\mu+\psi) a_{1}\right)(\mu+\psi)}, \\
I= & \frac{(\mu+\psi)^{2} a_{1}}{\left(e^{(\mu+\psi) t}(\alpha \psi+\mu \psi-\alpha \gamma \mu-\alpha \gamma \psi)-(\mu+\psi) a_{1}\right)(\alpha-\psi)} .
\end{aligned}
$$

Finally, if $\tilde{\beta}=\alpha-\psi$ and $\gamma=\frac{\psi(\alpha+\mu)}{\alpha(\mu+\psi)}$ then equation (2.10) can be immediately integrated, i.e.:

$$
u=\frac{1}{e^{(\mu+\psi) t} a_{1}+a_{2}},
$$

and consequently the general solution of (1.5)-(1.7) is

$$
\begin{aligned}
S & =\frac{\mu+\psi+\mu(\alpha-\psi)\left(e^{(\mu+\psi) t} a_{1}+a_{2}\right)}{\left(e^{(\mu+\psi) t} a_{1}+a_{2}\right)(\alpha-\psi)(\mu+\psi)} \\
V & =\frac{e^{(\mu+\psi) t}(\alpha-\psi) a_{1} \psi+\alpha a_{2} \psi+a_{2} \mu^{2}+2 a_{2} \mu \psi-\mu-\psi}{\left(e^{(\mu+\psi) t} a_{1}+a_{2}\right)(\alpha-\psi)(\mu+\psi)} \\
I & =\frac{-(\mu+\psi) a_{2}}{\left(e^{(\mu+\psi) t} a_{1}+a_{2}\right)(\alpha-\psi)}
\end{aligned}
$$




\section{Case (2)}

$$
\beta=\frac{(1-\gamma)(\mu+\psi) \alpha}{\mu}, \tilde{\beta}=\frac{(\alpha \gamma-\psi)(\mu+\psi)}{\psi}, \quad(\psi<\alpha \gamma)
$$

Equation (2.10) admits a non-abelian transitive two-dimensional Lie algebra generated by the following operators:

$$
\Gamma_{1}=e^{(\mu+\psi) t}\left(\partial_{t}-(\mu+\psi) u \partial_{u}\right), \quad \Gamma_{2}=\partial_{t}
$$

A basis of the differential invariants of order $\leq 1$ for operator $\Gamma_{1}$ in $(2.35)$ is

$$
\tilde{t}=u e^{(\mu+\psi) t}, \quad \tilde{u}=(\dot{u}+u \mu+u \psi) e^{2(\mu+\psi) t},
$$

and therefore equation (2.10) becomes the following first-order equation:

$$
\frac{\mathrm{d} \tilde{u}}{\mathrm{~d} \tilde{t}}=\frac{(-\alpha \gamma \mu+\alpha \gamma \psi-\alpha \psi+\mu \psi) \tilde{u}+(\alpha \gamma \mu+\alpha \gamma \psi-\alpha \psi-\mu \psi) \tilde{t}^{2}}{\alpha \psi(\gamma-1) \tilde{t}},
$$

which can be easily integrated, i.e.:

$$
\tilde{u}=a_{1} \tilde{t} \frac{(\alpha \gamma-\psi) \mu+(1-\gamma) \alpha \psi}{(1-\gamma) \alpha \psi}+\tilde{t}^{2} .
$$

The integration of (2.37) is not haphazard, but follows from Lie symmetry method itself [22]. In fact equation (2.37) admits the Lie symmetry generated by $\Gamma_{2}$ in $(2.35)$ which is transformed into

$$
\tilde{\Gamma}_{2}=(\mu+\psi)\left(\tilde{t} \partial_{\tilde{t}}+2 \tilde{u} \partial_{\tilde{u}}\right)
$$

by means of (2.36). Substitution of (2.36) into (2.38) yields the following first-order equation:

$$
\dot{u}=a_{1} u^{\frac{(\alpha \gamma-\psi) \mu}{(1-\gamma) \alpha \psi}+1} e^{\left(\frac{(\alpha \gamma-\psi) \mu}{(1-\gamma) \alpha \psi}-1\right)(\mu+\psi) t}+u(u-\psi-\mu),
$$

which is easy to integrate because it admits the Lie symmetry generated by $\Gamma_{1}$ in (2.35). In fact Lie proved that if one knows a symmetry $\tau(t, u) \partial_{t}+\xi(t, u) \partial_{u}$ of a first-order ordinary differential equation, say $\dot{u}=f(t, u)$, then an integrating factor for the corresponding linear differential form, say $\mathrm{d} u-f(t, u) \mathrm{d} t=0$, is $\frac{1}{\xi-f(t, u) \tau}[22]$. Thus

$$
\frac{-e^{-(\mu+\psi) t}}{a_{1} u^{\frac{(\alpha \gamma-\psi) \mu}{(1-\gamma) \alpha \psi}+1} e^{\left(\frac{(\alpha \gamma-\psi) \mu}{(1-\gamma) \alpha \psi}-1\right)(\mu+\psi) t}+u^{2}}
$$

is an integrating factor for the linear differential form corresponding to equation (2.40), i.e.:

$$
\mathrm{d} u-\left(a_{1} u^{\frac{(\alpha \gamma-\psi) \mu}{(1-\gamma) \alpha \psi}+1} e^{\left(\frac{(\alpha \gamma-\psi) \mu}{(1-\gamma) \alpha \psi}-1\right)(\mu+\psi) t}+u(u-\psi-\mu)\right) \mathrm{d} t=0
$$

Then the general solution of equation (2.10) could be derived by two quadratures although in implicit form. 


\section{Case (3)}

$$
\beta=2 \tilde{\beta}, \quad \gamma=\frac{(\alpha+2 \mu) \psi}{(2 \mu+\psi) \alpha}, \quad \tilde{\beta}=\frac{(\alpha-\psi)(\mu+\psi)}{2 \mu+\psi}
$$

Equation (2.10) admits the same two-dimensional Lie algebra as in Case (2). A basis of the differential invariants of order $\leq 1$ for operator $\Gamma_{1}$ in $(2.35)$ is given in $(2.36)$ and equation (2.10) becomes the following first-order equation:

$$
\frac{\mathrm{d} \tilde{u}}{\mathrm{~d} \tilde{t}}=\frac{3 \tilde{u}+\tilde{t}^{2}}{2 \tilde{t}}
$$

which can be easily integrated, i.e.:

$$
\tilde{u}=a_{1} \tilde{t} \sqrt{\tilde{t}}+\tilde{t}^{2} .
$$

Substitution of (2.36) into (2.44) yields the following first-order equation:

$$
\dot{u}=-a_{1} u \sqrt{u} e^{-(\mu+\psi) t / 2}+u(u-\mu-\psi),
$$

which is easy to integrate because it admits the Lie symmetry generated by $\Gamma_{1}$ in (2.35), i.e.:

$\frac{2}{a_{1} \sqrt{u}} e^{-(\mu+\psi) t / 2}-\frac{e^{-(\mu+\psi) t}}{\mu+\psi}+\frac{1}{a_{1}^{2}} \log \left(u e^{(\mu+\psi) t}\right)-\frac{2}{a_{1}^{2}} \log \left(\sqrt{u} e^{(\mu+\psi) t / 2}+a_{1}\right)=a_{2}$.

This solution is in implicit form and as such we cannot derive the general solution of (1.5)-(1.7) in explicit form.

\section{Case (4)}

$$
\tilde{\beta}=\alpha \gamma+\mu, \quad \gamma=\frac{(2 \mu+\psi) \psi}{\alpha \mu}, \quad \beta=\frac{-(2 \mu+\psi) \psi+\alpha \mu}{\mu^{2}}(\mu+\psi)
$$

Equation (2.10) admits the same two-dimensional Lie algebra as in Case (2). A basis of the differential invariants of order $\leq 1$ for operator $\Gamma_{1}$ in (2.35) is given in (2.36) and equation (2.10) becomes the following first-order equation:

$$
\frac{\mathrm{d} \tilde{u}}{\mathrm{~d} \tilde{t}}=\frac{\left(\alpha \mu+\mu^{2}-\mu \psi-\psi^{2}\right) \tilde{u}+\left(\alpha \mu-\mu^{2}-3 \mu \psi-\psi^{2}\right) \tilde{t}^{2}}{\left(\alpha \mu-2 \mu \psi-\psi^{2}\right) \tilde{t}},
$$

which can be easily integrated, i.e.:

$$
\tilde{u}=a_{1} \tilde{t} \frac{-\alpha \mu+\psi^{2}-\mu^{2}+\mu \psi}{2 \mu \psi+\psi^{2}-\alpha \mu}+\tilde{t}^{2} .
$$

Substitution of (2.36) into (2.48) yields the following first-order equation:

$$
\dot{u}=a_{1} e^{-t \frac{(\mu+\psi)\left(\alpha \mu-\psi^{2}-\mu^{2}-3 \mu \psi\right)}{-2 \mu \psi-\psi^{2}+\alpha \mu}} u^{\frac{\alpha \mu-\psi^{2}+\mu^{2}-\mu \psi}{-2 \mu \psi-\psi^{2}+\alpha \mu}}+u(u-\mu-\psi),
$$

which could be integrated although in implicit form because it admits the Lie symmetry generated by $\Gamma_{1}$ in (2.35). As in the previous two cases the general solution of (1.5)-(1.7) cannot be derived in explicit form. 


\section{Case (5)}

$$
\tilde{\beta}=0, \quad \psi=\alpha \gamma, \quad \beta=\frac{\alpha(1-\gamma)(\alpha \gamma+\mu)}{\mu}
$$

Equation (2.10) admits the same two-dimensional Lie algebra as in Case (2), i.e.:

$$
\Gamma_{1}=e^{(\mu+\alpha \gamma) t}\left(\partial_{t}-(\mu+\alpha \gamma) u \partial_{u}\right), \quad \Gamma_{2}=\partial_{t}
$$

A basis of the differential invariants of order $\leq 1$ for operator $\Gamma_{1}$ in $(2.50)$ is

$$
\tilde{t}=u e^{(\mu+\alpha \gamma) t}, \quad \tilde{u}=(\dot{u}+u \mu+u \alpha \gamma) e^{2(\mu+\alpha \gamma) t},
$$

and therefore equation (2.10) becomes the following first-order equation:

$$
\frac{\mathrm{d} \tilde{u}}{\mathrm{~d} \tilde{t}}=\frac{\tilde{u}+\tilde{t}^{2}}{\tilde{t}}
$$

which can be easily integrated, i.e.:

$$
\tilde{u}=a_{1} \tilde{t}+\tilde{t}^{2} .
$$

The integration of (2.52) is not haphazard, but follows from Lie symmetry method itself [22] as stated in Case (2). Substitution of (2.51) into (2.53) yields the following first-order equation:

$$
\dot{u}=\frac{u\left(-e^{(\alpha \gamma+\mu) t}(\alpha \gamma+\mu-u)+a_{1}\right)}{e^{(\alpha \gamma+\mu) t}}
$$

which is easy to integrate because it admits the Lie symmetry generated by $\Gamma_{1}$ in $(2.50)$. Thus the general solution of (2.10) is

$$
u=\frac{a_{1}}{e^{(\alpha \gamma+\mu) t}\left(e^{a_{1} /\left(e^{(\alpha \gamma+\mu) t}(\alpha \gamma+\mu)\right.} a_{1} a_{2}-1\right)} .
$$

and the general solution of (1.5)-(1.7) is:

$$
\begin{aligned}
S & =\frac{\left(e^{(\alpha \gamma+\mu) t}\left(e^{a_{1} /\left(e^{(\alpha \gamma+\mu) t}(\alpha \gamma+\mu)\right)} a_{1} a_{2}-1\right)(\gamma-1) \alpha-a_{1}\right) \mu}{e^{(\alpha \gamma+\mu) t}\left(e^{a_{1} /\left(e^{(\alpha \gamma+\mu) t}(\alpha \gamma+\mu)\right)} a_{1} a_{2}-1\right)(\alpha \gamma+\mu)(\gamma-1) \alpha} \\
V & =\frac{e^{(\alpha \gamma+\mu) t} \alpha^{2} \gamma(\gamma-1)-a_{1} \mu}{e^{(\alpha \gamma+\mu) t}(\alpha \gamma+\mu)(\gamma-1) \alpha} \\
I & =\frac{e^{a_{1} /\left(e^{(\alpha \gamma+\mu) t}(\alpha \gamma+\mu)\right)} a_{1}^{2} a_{2} \mu}{e^{(\alpha \gamma+\mu) t}\left(e^{a_{1} /\left(e^{(\alpha \gamma+\mu) t}(\alpha \gamma+\mu)\right)} a_{1} a_{2}-1\right)(\alpha \gamma+\mu)(\gamma-1) \alpha}
\end{aligned}
$$

There exist other cases such as

$$
\beta=\frac{[(1-\gamma) \alpha-2(\mu+\psi)] \tilde{\beta}}{\tilde{\beta}-\mu-\alpha \gamma}
$$

that we do not consider because they give rise to unacceptable conditions on parameters, e.g. $\tilde{\beta}=-\beta$. 


\section{Discussion}

We now study in detail the solutions in closed form that we have obtained in cases (1) and (5). We simulate the effectiveness of a disease management program in the core group by plotting the solutions with the help of the graphing capability of MAPLE. Common sense would dictate that if the transmission rates are low and the education rate is high, then both the number of susceptibles and infecteds will decrease leading to a successful disease management program. However, our analysis singles out several different events, such as an astonishing one in which at high transmission rate and high vaccination/education rate the number of infecteds decreases if the initial prevalence of infecteds is high, but increases if it is low! Also, in some instances a temporary increase of either the number of infecteds or susceptibles followed by a decrease (quite sharp in certain settings) occurs despite the presence of the hight vaccination/education rate and low transmission rate. Note that the qualitative description by Hadeler and Castillo-Chavez is limited to the study of equilibrium points, their stability and bifurcation diagrams.

We use the same numerical value of $\alpha, \mu$ and $\gamma$ as given in [8], i.e.:

$$
\alpha=4, \quad \mu=0.2, \quad \gamma=0.025 .
$$

The numerical value of the other parameters are derived from the relationships that Lie group analysis has discerned.

We simulate the dynamics of the core group taking into consideration two different initial conditions at time $t=0$ :

(A) No vaccinated/educated are present and the prevalence of infecteds is relatively small, i.e. $S(0)=0.9, V(0)=0, I(0)=0.1$

(B) No vaccinated/educated are present and the prevalence of infecteds is high being nearly half the size of the core group, i.e. $S(0)=0.6, V(0)=0, I(0)=0.4$

In all the figures, the solid line represents the plot of $S$, the lighter dashed line represents the plot of $V$, and the darker dashed line represents the plot of $I$.

$$
\begin{gathered}
\text { Case (1) } \\
\beta=\tilde{\beta}
\end{gathered}
$$

In this case the transmission rate between infecteds and susceptibles $\beta$ and between infecteds and educated/vaccinated $\tilde{\beta}$ is the same. We simulate the dynamics in the core group by assuming three different values of $\tilde{\beta}$ and three different values of $\psi$ within the range considered in [8], namely

$$
\psi=0.1\left\{\begin{array}{l}
\tilde{\beta}=0.3 \\
\tilde{\beta}=3.3 \\
\tilde{\beta}=6
\end{array} \quad \psi=1\left\{\begin{array}{l}
\tilde{\beta}=0.3 \\
\tilde{\beta}=3.3 \\
\tilde{\beta}=6
\end{array} \quad \psi=2\left\{\begin{array}{l}
\tilde{\beta}=0.3 \\
\tilde{\beta}=3.3 \\
\tilde{\beta}=6
\end{array}\right.\right.\right.
$$

In Fig. 1-A1 and in Fig. 1-B1 we show the dynamics in the core group if $\psi=0.1, \tilde{\beta}=$ 0.3 and in correspondence of the two initial conditions (A) and (B), respectively. In Fig. $1-\mathrm{A} 2$ and in Fig. $1-\mathrm{B} 2$ we show the dynamics in the core group if $\psi=0.1, \tilde{\beta}=3.3$ 


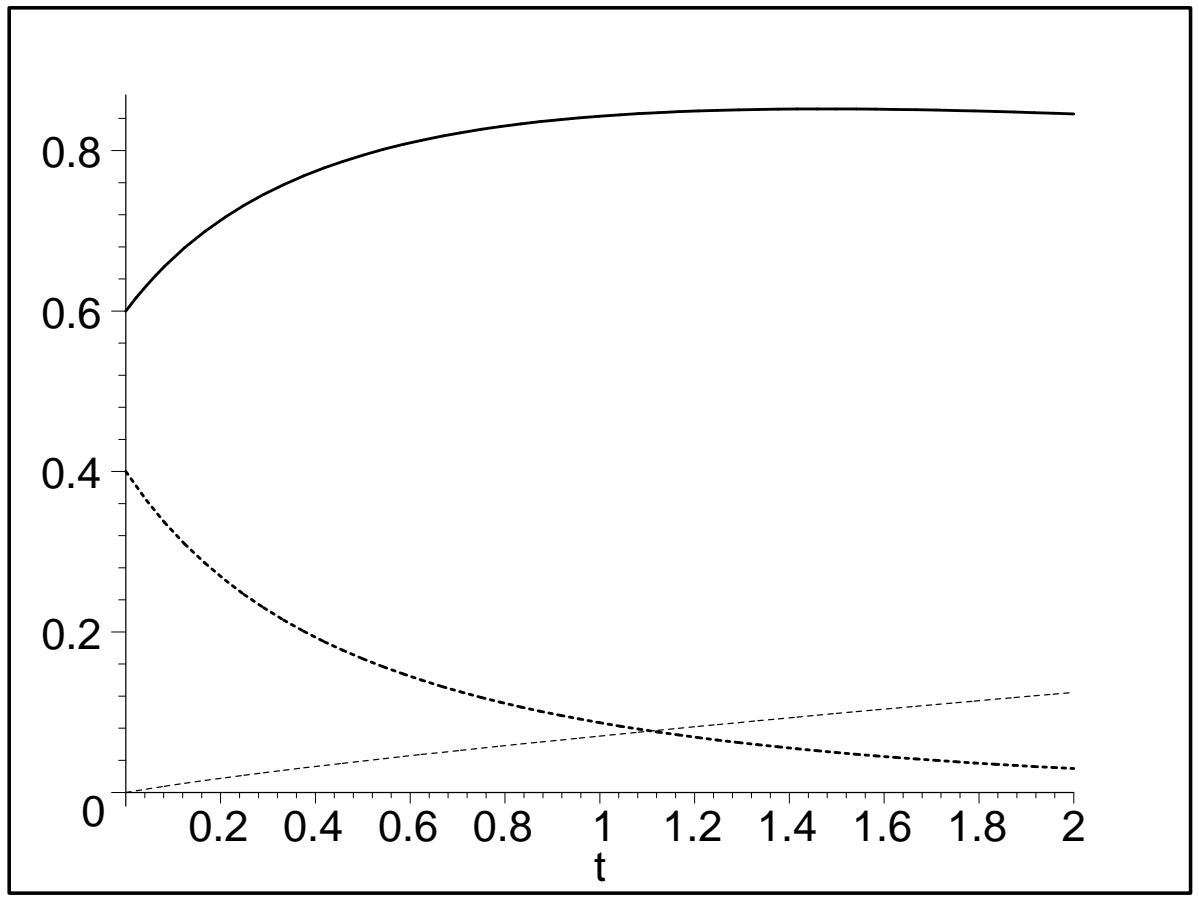

Fig. 1-B2: $\beta=\tilde{\beta}=3.3, \psi=0.1, S(0)=0.6, V(0)=0, I(0)=0.4$

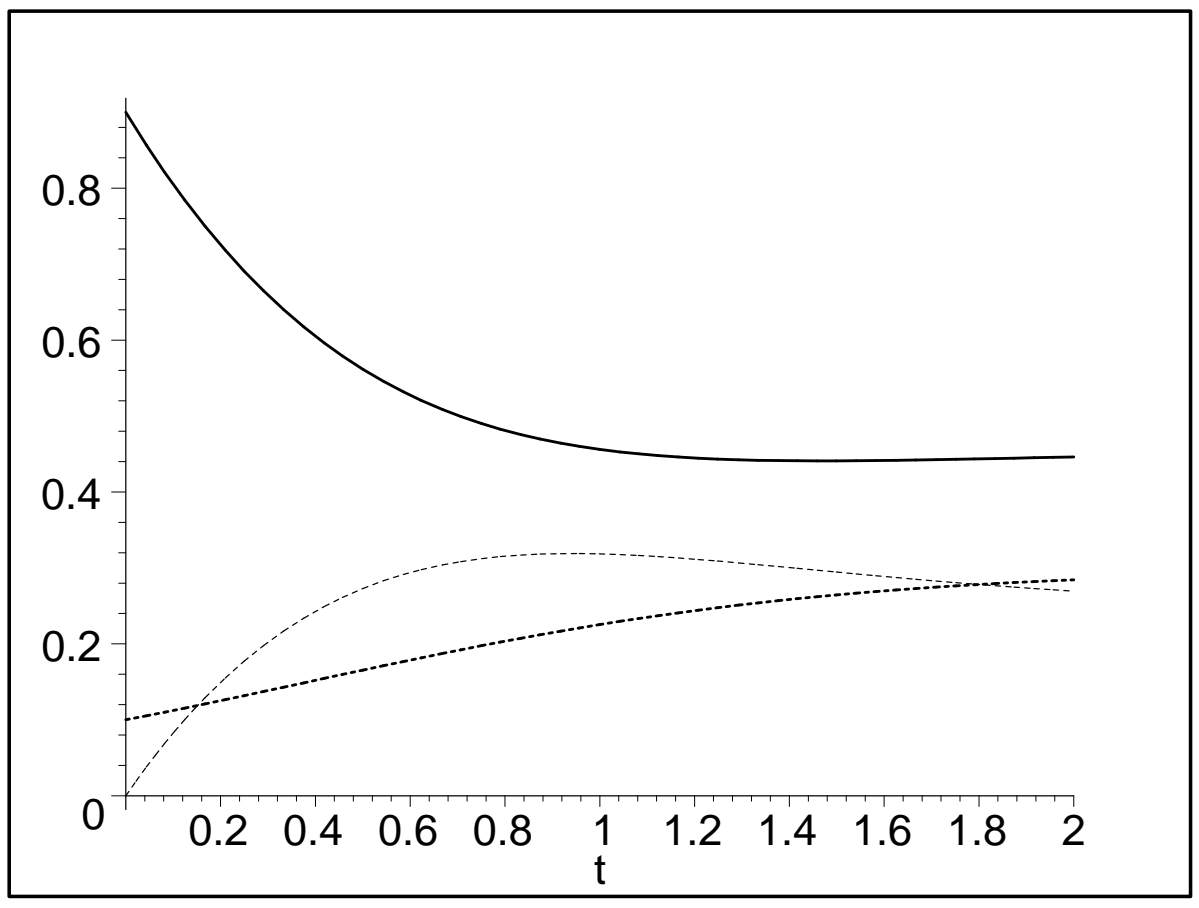

Fig. 1-A6: $\beta=\tilde{\beta}=6, \psi=1, S(0)=0.9, V(0)=0, I(0)=0.1$ 
and corresponding to each of the two initial conditions (A) and (B), respectively. In Fig. 1-A3 and in Fig. 1-B3 we show the dynamics in the core group if $\psi=0.1, \tilde{\beta}=6$ and corresponding to each of the two initial conditions (A) and (B), respectively. In Fig. 1A4 and in Fig. 1-B4 we show the dynamics in the core group if $\psi=1, \tilde{\beta}=0.3$ and corresponding to each of the two initial conditions (A) and (B), respectively. In Fig. 1A5 and in Fig. 1-B5 we show the dynamics in the core group if $\psi=1, \tilde{\beta}=3.3$ and corresponding to each of the two initial conditions (A) and (B), respectively. In Fig. 1-A6 and in Fig. 1-B6 we show the dynamics in the core group if $\psi=1, \tilde{\beta}=6$ and corresponding to each of the two initial conditions (A) and (B), respectively. In Fig. 1-A7 and in Fig. 1-B7 we show the dynamics in the core group if $\psi=2, \tilde{\beta}=0.3$ and corresponding to each of the two initial conditions (A) and (B), respectively. In Fig. 1-A8 and in Fig. 1-B8 we show the dynamics in the core group if $\psi=2, \tilde{\beta}=3.3$ and corresponding to each of the two initial conditions (A) and (B), respectively. In Fig. 1-A9 and in Fig. 1-B9 we show the dynamics in the core group if $\psi=2, \tilde{\beta}=6$ and corresponding to each of the two initial conditions (A) and (B), respectively. We notice the following different outputs of the vaccination/education program:

Complete Success, i.e. both number of susceptibles and infected decrease, is obtained in the following cases:

- the transmission rates are either low or in the middle range, the education/vaccination rate is in the middle range, and the initial prevalence of infecteds is low (Fig. 1-A4), (Fig. 1-A5);

- the transmission rates are high, the education/vaccination rate is in the middle range, and the initial prevalence of infecteds is high (Fig. 1-B6);

- the transmission rates are low, the education/vaccination rate is high, and the initial prevalence of infecteds is low (Fig. 1-A7);

- the transmission rates are in the middle range, and the education/vaccination rate is high, regardless of the initial prevalence of infecteds (Fig. 1-A8), (Fig. $1-\mathrm{B} 8)$;

- the transmission rates are high, the education/vaccination rate is high, and the initial prevalence of infecteds is high (Fig. 1-B9).

Increase of number of susceptibles followed by decrease, while the number of infecteds decreases immediately, is obtained in the following cases:

- the transmission rates are low, the education/vaccination rate is low, regardless of the initial prevalence of infecteds (Fig. 1-A1), (Fig. 1-B1);

- the transmission rates are in the middle range, the education/vaccination rate is low, and the initial prevalence of infecteds is low (Fig. 1-A2);

- the transmission rates are either low or in the middle range, the education/vaccination rate is in the middle range, and the initial prevalence of infecteds is high (Fig. 1-B4), (Fig. 1-B5);

- the transmission rates are low, the education/vaccination rate is high, and the initial prevalence of infecteds is high (Fig. 1-B7); 


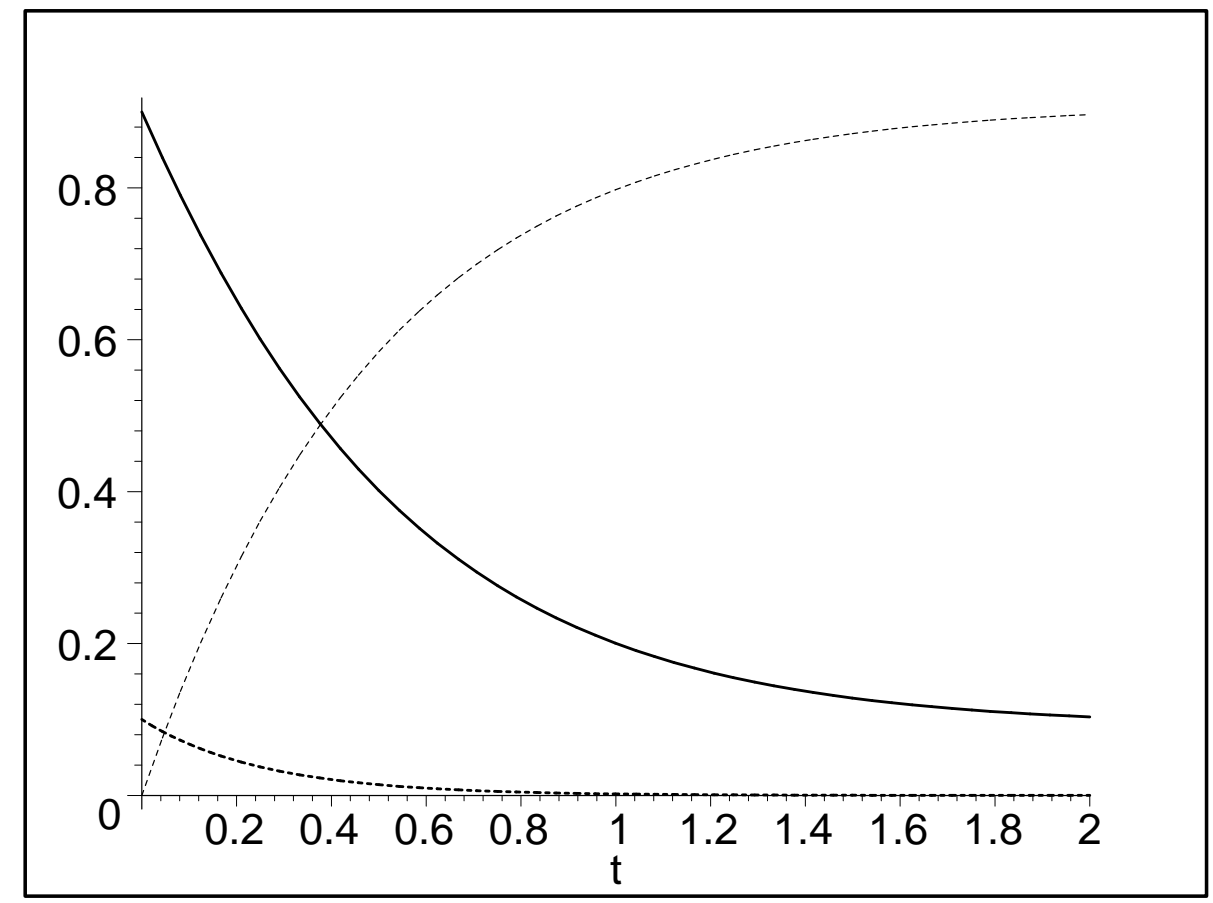

Fig. 1-A7: $\beta=\tilde{\beta}=0.3, \psi=2, S(0)=0.9, V(0)=0, I(0)=0.1$

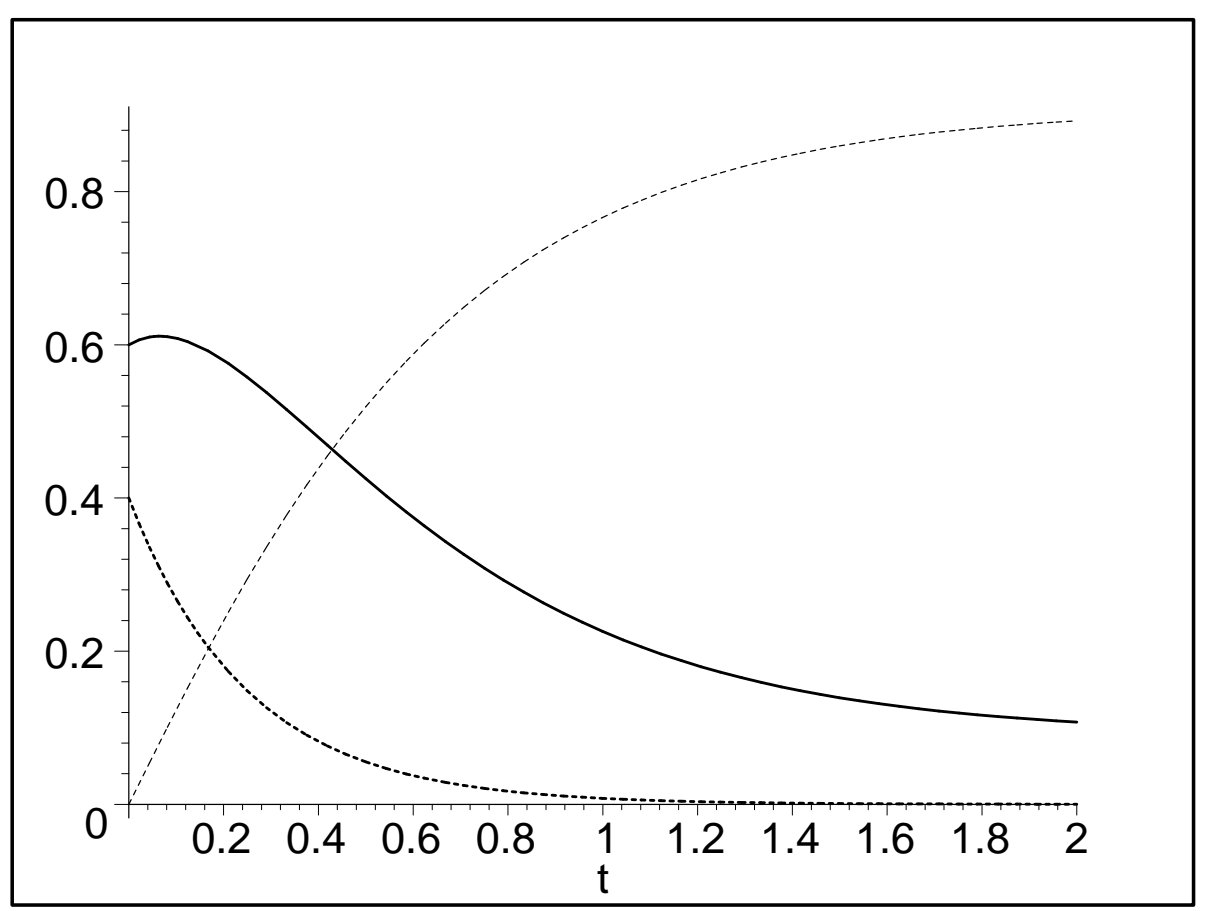

Fig. 1-B7: $\beta=\tilde{\beta}=0.3, \psi=2, S(0)=0.6, V(0)=0, I(0)=0.4$ 


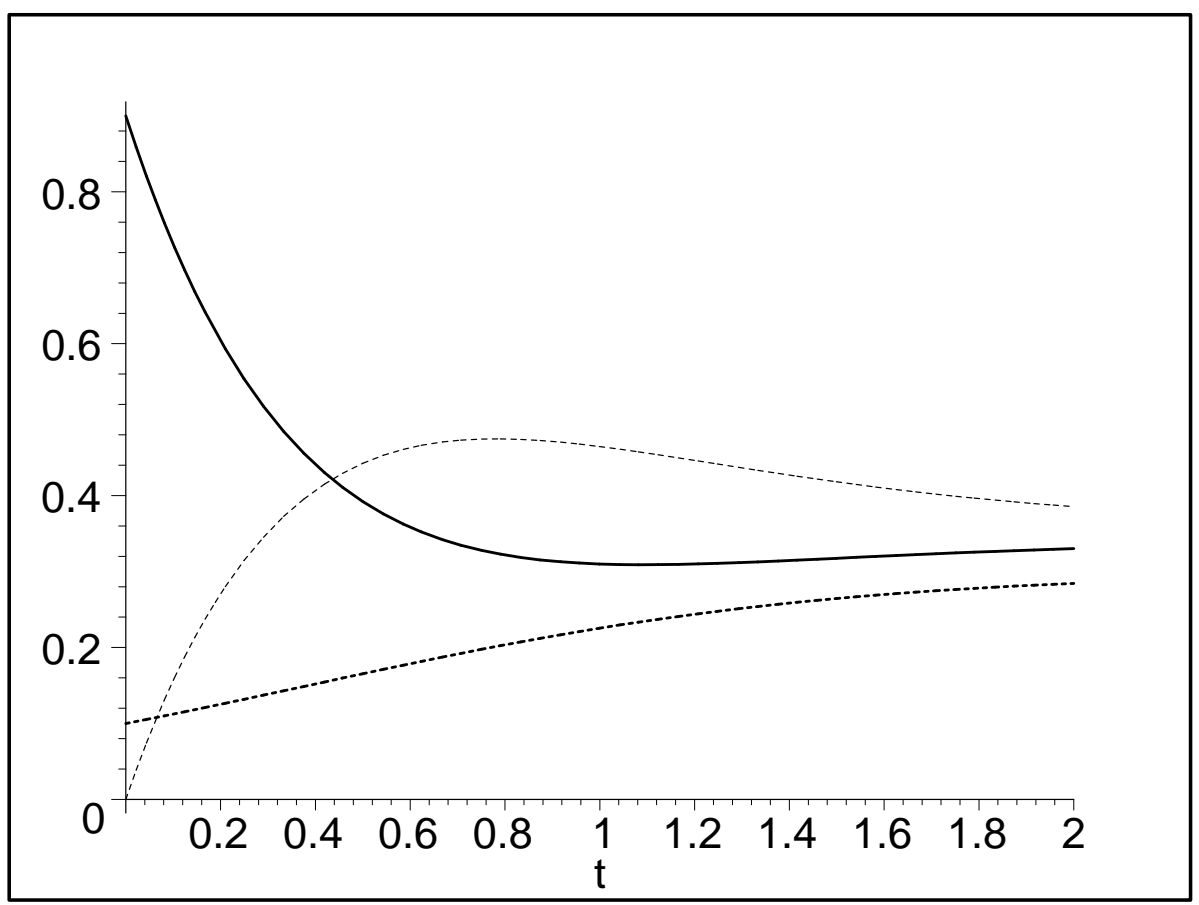

Fig. 1-A9: $\beta=\tilde{\beta}=6, \psi=2, S(0)=0.9, V(0)=0, I(0)=0.1$

Increase of number of susceptibles to a steady state, while the number of infecteds decreases immediately, is obtained in the following cases:

- the transmission rates are either in the middle range or high, the education/vaccination rate is low, and the initial prevalence of infecteds is high (Fig. 1-B2), (Fig. 1-B3);

Increase of number of infecteds followed by decrease, while the number of susceptibles decreases immediately, is obtained in the following case:

- the transmission rates are high, the education/vaccination rate is in the middle range, and the initial prevalence of infecteds is low (Fig. 1-A6);

Increase of number of infecteds, while the number of susceptibles decreases immediately, is obtained in the following cases:

- the transmission rates are high, the education/vaccination rate is either low or high, and the initial prevalence of infecteds is low (Fig. 1-A3), (Fig. 1-A9).

In summary, the worst scenario occurs when the initial prevalence of infecteds is low. This paradoxical behavior has already been noticed in [8] and [6], but not with the same quantitative details that we have shown here, thanks to Lie group analysis. 


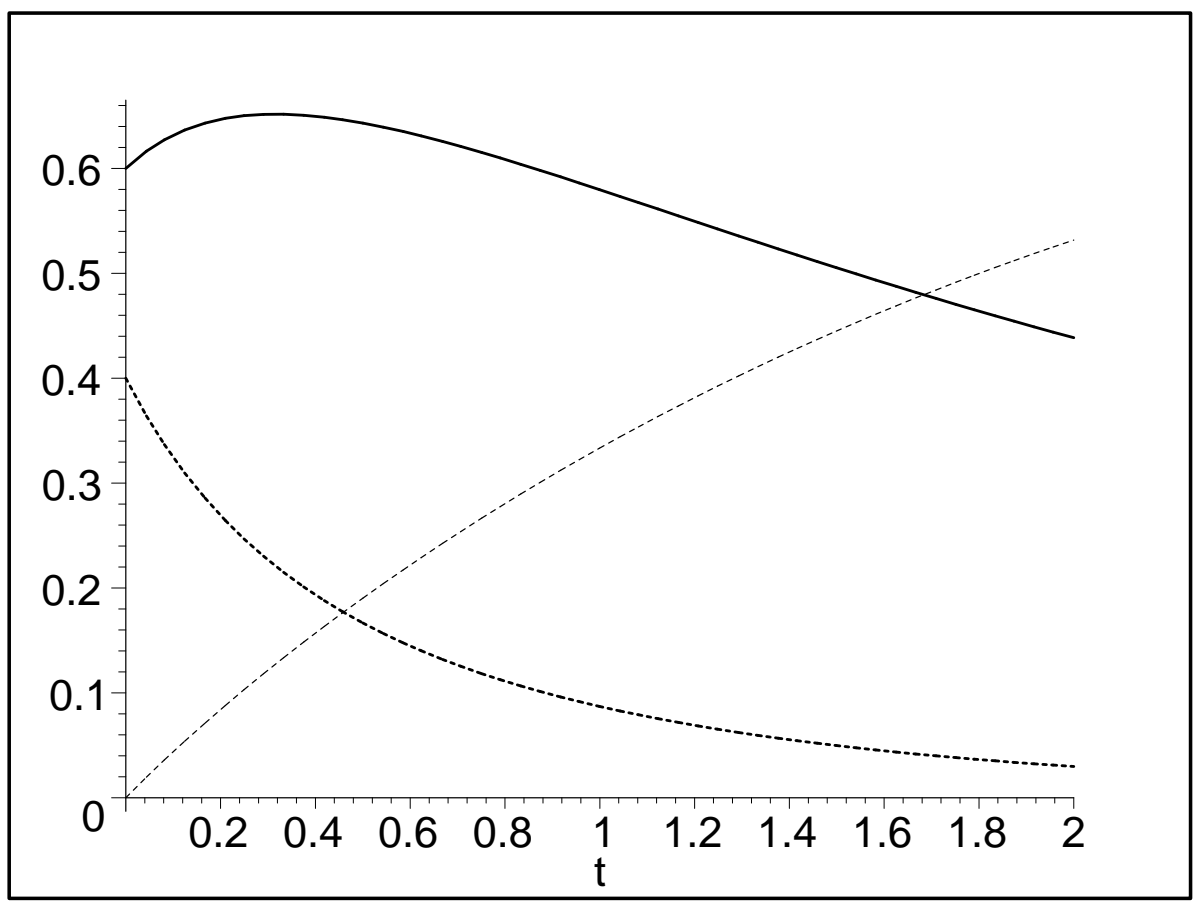

Fig. 1s1-B1: $\beta=\tilde{\beta}=3.3, \psi=0.7, S(0)=0.6, V(0)=0, I(0)=0.4$

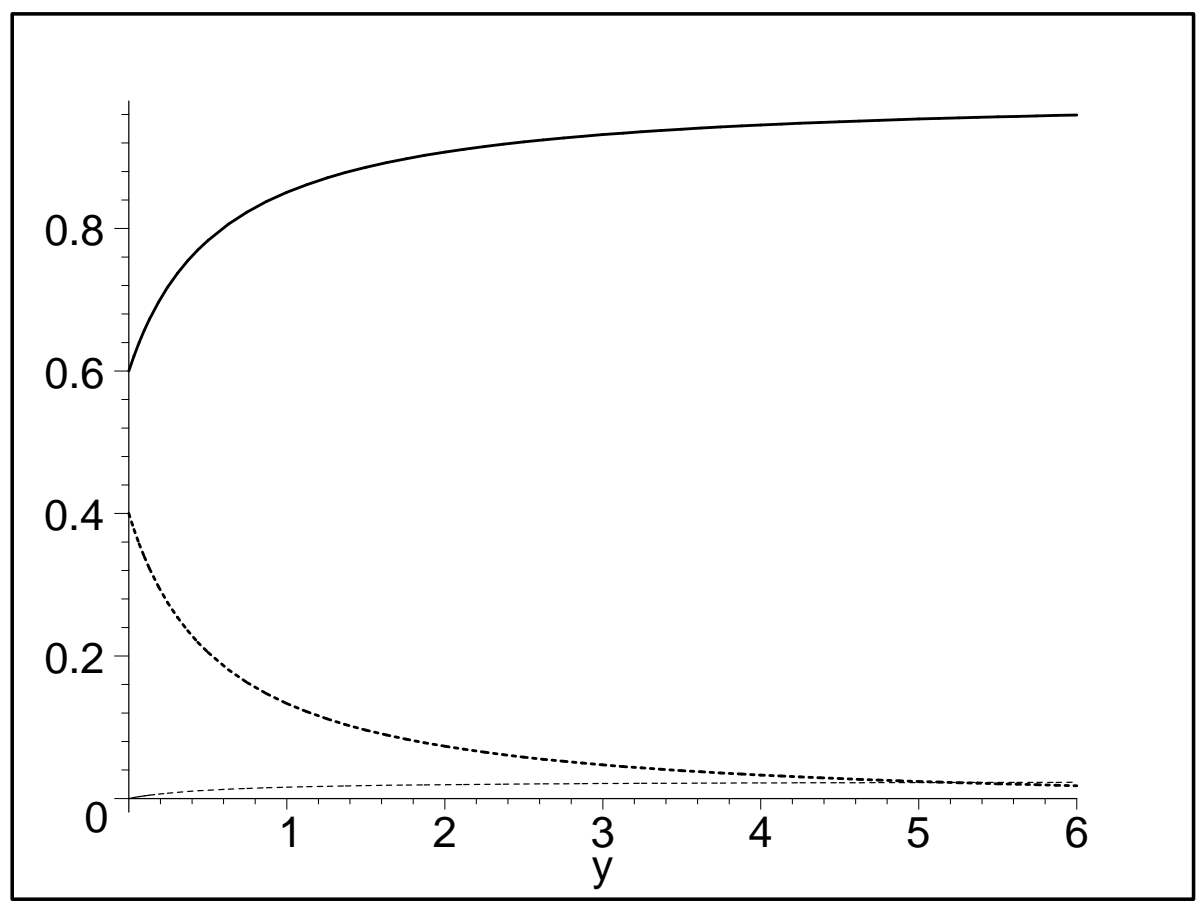

Fig. 1s2-B: $\beta=\tilde{\beta}=3.9951, \psi=0.004878, S(0)=0.6, V(0)=0, I(0)=0.4$ 
In the case $\tilde{\beta}=\alpha-\psi=4-\psi$ we simulate the dynamics of the core group by assuming three different values for $\psi$, which correspond to values of $\tilde{\beta}$ considered in [8] namely

$$
\psi=0.7(\tilde{\beta}=3.3), \quad \psi=2.2(\tilde{\beta}=1.8), \quad \psi=3.7(\tilde{\beta}=0.3) .
$$

In Fig. 1s1-A1 and Fig. 1s1-B1 we show the dynamics in the core group if $\psi=0.7(\tilde{\beta}=$ 3.3 ) and corresponding to each of the two initial conditions (A) and (B), respectively. In Fig. 1s1-A2 and Fig. 1s1-B2 we show the dynamics in the core group if $\psi=2.2(\tilde{\beta}=1.8)$ and corresponding to each of the two initial conditions (A) and (B), respectively. In Fig. 1s1-A3 and Fig. 1s1-B3 we show the dynamics in the core group if $\psi=3.7(\tilde{\beta}=0.3)$ and corresponding to each of the two initial conditions (A) and (B), respectively. One can obviously notice that the smaller the transmission rate (the higher the education/vaccination rate) the steeper the increase of the number of educated/vaccinated (Fig. 1s1-A1, Fig. 1s1-A2, Fig. 1s1-B2, Fig. 1s1-A3, Fig. 1s1-B3). Less obvious is the temporary increase of the number of susceptibles followed by a decrease in the case of a relatively high initial prevalence of infecteds $(I(0)=0.4)$ and a relatively high transmission rate $(\tilde{\beta}=3.3)$ as seen in Fig. 1s1-B1.

If $\tilde{\beta}=\alpha-\psi$ and $\gamma=\frac{\psi(\alpha+\mu)}{\alpha(\mu+\psi)}$ which imply ${ }^{2}$

$$
\psi=0.004878, \quad \tilde{\beta}=3.9951
$$

then in Fig. 1s2-A and Fig. 1s2-B we show the dynamics in the core group corresponding to each of the two initial conditions (A) and (B), respectively. Note that the education/vaccination rate is very small $(\psi=0.004878)$. If the initial prevalence of infecteds is high $(I(0)=0.4)$ then the number of susceptibles increases up to a steady state as seen in Fig. 1s2-B. Note that the time at which the steady state is reached can be exactly calculated from the solution (2.32). If the initial prevalence of infecteds is small $(I(0)=0.1)$ then the dynamics is nearly steady, as seen in Fig. 1s2-A.

\section{Case (5)}

$$
\tilde{\beta}=0, \quad \psi=\alpha \gamma=0.1, \quad \beta=\frac{\alpha(1-\gamma)(\alpha \gamma+\mu)}{\mu}=5.85
$$

In this case of high transmission rate and low vaccination/education rate, it is noteworthy that if the initial prevalence of infecteds is small $(I(0)=0.1)$, then there is a delay in the effectiveness of the vaccination/education program in targeting the infecteds as can be seen in Fig. 5-A, while if the initial prevalence of infecteds is high $(I(0)=0.4)$, then the vaccination/education program is immediately effective with the infecteds but has no effect on the susceptibles (Fig. 5-B) who slightly increase to a steady state.

\section{Final remarks}

We were able to find that system (1.5-1.7) possesses enough Lie symmetries to yield integration by quadrature if certain relationships among the involved parameters are imposed. In particular, if $\beta=\tilde{\beta}$, system (1.5-1.7) is actually linearizable, namely it can be transformed into a linear equation of second order. We would like to add that, although in

\footnotetext{
${ }^{2}$ We have allowed MAPLE to use up to five digits when calculating with software floating-point numbers.
} 


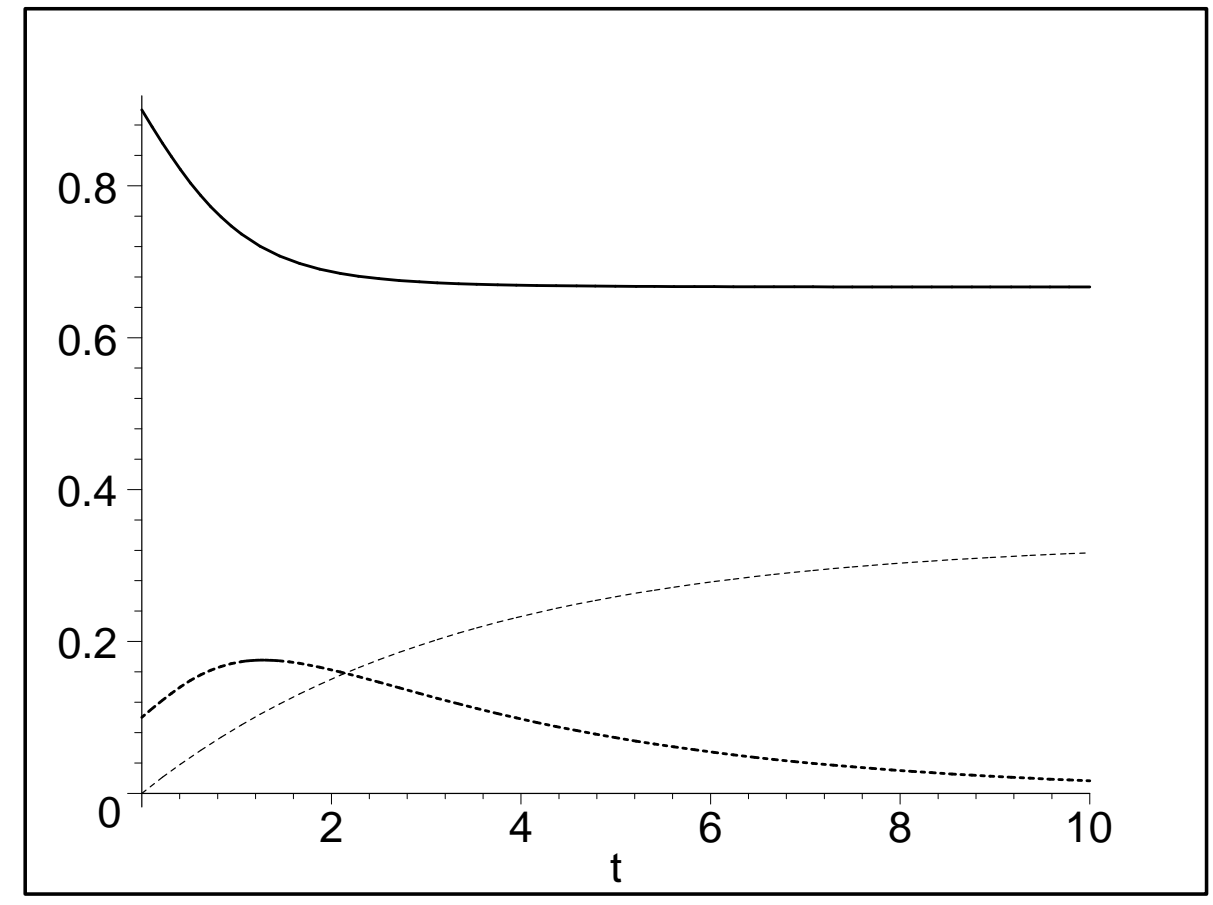

Fig. 5-A: $\tilde{\beta}=0, \psi=0.1, \beta=5.85, S(0)=0.9, V(0)=0, I(0)=0.1$

some cases we were unable to write down the solution in closed form, first integrals were explicitly derived such as (2.40), (2.45), (2.49), (2.54). This raises the question of what is the epidemiological meaning of conservation laws. They may also be good candidates for Lyapunov's functions. Also we have shown that Lie group analysis gives deeper quantitative insight into the dynamics of epidemics. We have studied the dynamics of the core group by plotting the obtained solutions. One could easily perform an analytical study; for example, calculate for which values of the involved parameters the number of infecteds increases instead of decreasing, or the number of susceptibles increases instead of decreasing, or the number of vaccinated/educated decreases instead of increasing. It is obvious that if the general solution of a mathematical model is obtained, then all the mathematical properties of that model are trivially revealed.

It is a well-know fact that when Lie group analysis is applied to physical models then integrable cases can be found (e.g., [23], [28]). The same is true in the case of epidemiological models [37],[31], although the meaning of integrability is somewhat blurred in this context. One can easily object that our cases correspond to very particular values of the parameters, and therefore Lie group analysis will give you a limited number of possible cases. Nevertheless we may reply that very little is known about those parameters a priori, and that nature has shown (e.g., the ubiquitous presence of Fibonacci numbers in biology) a predisposition to favor certain values more than others.

In the Introduction to his Principia [24], [5], Newton stated

I wish we could derive the rest of the phenomena of nature by the same kind 
of reasoning from mechanical principles.

However, Pulte [34] has reminded us that in his lectures on analytical mechanics Jacobi said [16]:

Wherever Mathematics is mixed up with anything, which is outside its field, you will find attempts to demonstrate these merely propositions a priori, and it will be your task to find out the false deduction in each case...

Mathematics cannot invent how the relations of system of points depend on each other.

\section{References}

[1] Ames W F, Nonlinear Partial Differential Equations in Engineering. Vol. 2, Academic Press, New York, 1972.

[2] Bluman G W and Anco S, Symmetry and Integration Methods for Differential Equations, Springer-Verlag, New York, 2002.

[3] Bluman G W and Cole J D, Similarity Methods for Differential Equations, Springer-Verlag, New York, 1974.

[4] Bluman G W and Kumei S, Symmetries and Differential Equations, Springer-Verlag, New York, 1989.

[5] Cajori F, Sir Isaac Newton's Mathematical Principles of Natural Philosophy and His System of the World, University of California Press, Berkeley, 1946.

[6] Fineberg H V, Education to prevent AIDS: prospects and obstacles, Science 239 (1988), $592-596$.

[7] GonzÁlez-Gascón F and GonzÁlez-López A, Symmetries of differential equations. IV, J. Math. Phys. 24 (1983), 2006-2021

[8] Hadeler K P and Castillo-Chavez C, A core group model for disease transmission, Math. Biosci. 128 (1995), 41-55.

[9] Hawkins T, Jacobi and the birth of Lie's theory of groups, Arch. Hist. Exact Sciences 42 (1991), 187-278.

[10] Hawkins T, The Emergence of the Theory of Lie Groups: An Essay in the History of Mathematics 1869-1926, Springer-Verlag, New York, 2000.

[11] Hill J M, Differential Equations and Group Methods for Scientists and Engineers, CRC Press, Boca Raton, 1992.

[12] Hydon P E, Symmetry Methods for Differential Equations: A Beginner's Guide, Cambridge University Press, Cambridge, 2000.

[13] Ibragimov N H, CRC Handbook of Lie Group Analysis of Differential Equations, Vol. I: Symmetries, Exact Solutions, and Conservation Laws, CRC Press, Boca Raton, 1994.

[14] Ibragimov N H, CRC Handbook of Lie Group Analysis of Differential Equations, Vol. II: Applications in Engineering and Physical Sciences, CRC Press, Boca Raton, 1995. 
[15] Ibragimov N H, Elementary Lie Group Analysis and Ordinary Differential Equations, Wiley, New York, 1999.

[16] JACOBI C G J, Vorlesungen über Analytische Mechanik (1847-1848), Editor: Pulte H, Braunschweig/Wiesbaden, Vieweg, 1996.

[17] Leach P G L and NucCi M C, Reduction of the classical MICZ-Kepler problem to a twodimensional linear isotropic harmonic oscillator, J. Math. Phys. 45 (2004), 3590-3604.

[18] LiE S, Theorie der Transformationsgruppen. Erster Abschnitt, B G Teubner, Leipzig, 1888.

[19] LiE S, Theorie der Transformationsgruppen. Zweiter Abschnitt, B G Teubner, Leipzig, 1890.

[20] LiE S, Theorie der Transformationsgruppen. Dritter und Letzter Abschnitt, B G Teubner, Leipzig, 1893.

[21] LIE S, Geometrie der Berührungstransformationen, B G Teubner, Leipzig, 1896.

[22] S. LIE, Vorlesungen über Differentialgleichungen mit Bekannten Infinitesimalen Transformationen, B G Teubner, Leipzig, 1912.

[23] Marcelli M and NuCCi M C, Lie point symmetries and first integrals: the Kowalevsky top, J. Math. Phys. 44 (2003), 2111-2132.

[24] Newton I, Philosophiæ Naturalis Principia Mathematica, J Streater, London, 1687.

[25] NUCCI M C, Interactive REDUCE programs for calculating classical, non-classical and LieBäcklund symmetries of differential equations, Preprint Georgia Tech Math 062090-051 (1990)

[26] NUCCI M C, Interactive REDUCE programs for calculating Lie point, non-classical, LieBäcklund, and approximate symmetries of differential equations: manual and floppy disk, in CRC Handbook of Lie Group Analysis of Differential Equations. Vol. 3: New Trends in Theoretical Developments and Computational Methods, Editor: Ibragimov N H, CRC Press, Boca Raton, 1996, 415-481.

[27] Nucci M C, The complete Kepler group can be derived by Lie group analysis, J. Math. Phys. 37 (1996), 1772-1775.

[28] NuCCI M C, Lorenz integrable system moves à la Poinsot, J. Math. Phys., 44 (2003), 41074118.

[29] NuCCI M C, Calogero's goldfish is indeed a school of free particles, J. Phys. A: Math. Gen. 37 (2004), 11391-11400.

[30] NucCi M C and Leach P G L, The harmony in the Kepler and related problems, J. Math. Phys. 42 (2001), 746-764.

[31] Nucci M C and Leach P G L, An integrable S-I-S model, J. Math. Anal. Appl. 290 (2004), $506-518$.

[32] Olver P J, Applications of Lie Groups to Differential Equations, Springer-Verlag, New York, 1993.

[33] Ovsjannikov L V, Group Analysis of Differential Equations, Academic Press, New York, 1982. 
[34] Pulte H, Jacobi's criticism of Lagrange: the changing role of mathematics in the foundations of classical mechanics, Historia Math. 25 (1998), 154-184.

[35] Rogers C and Ames W F, Nonlinear Boundary Value Problems in Science and Engineering, Academic Press, New York, 1989.

[36] Stephani H, Differential Equations. Their Solution Using Symmetries, Cambridge University Press, Cambridge, 1989.

[37] TorRISI V and NuCCI M C, Application of Lie group analysis to a mathematical model which describes HIV transmission, in The Geometrical Study of Differential Equations, Editors: Leslie J A and Hobart T P, American Mathematical Society, Providence, 2001, 11-20. 


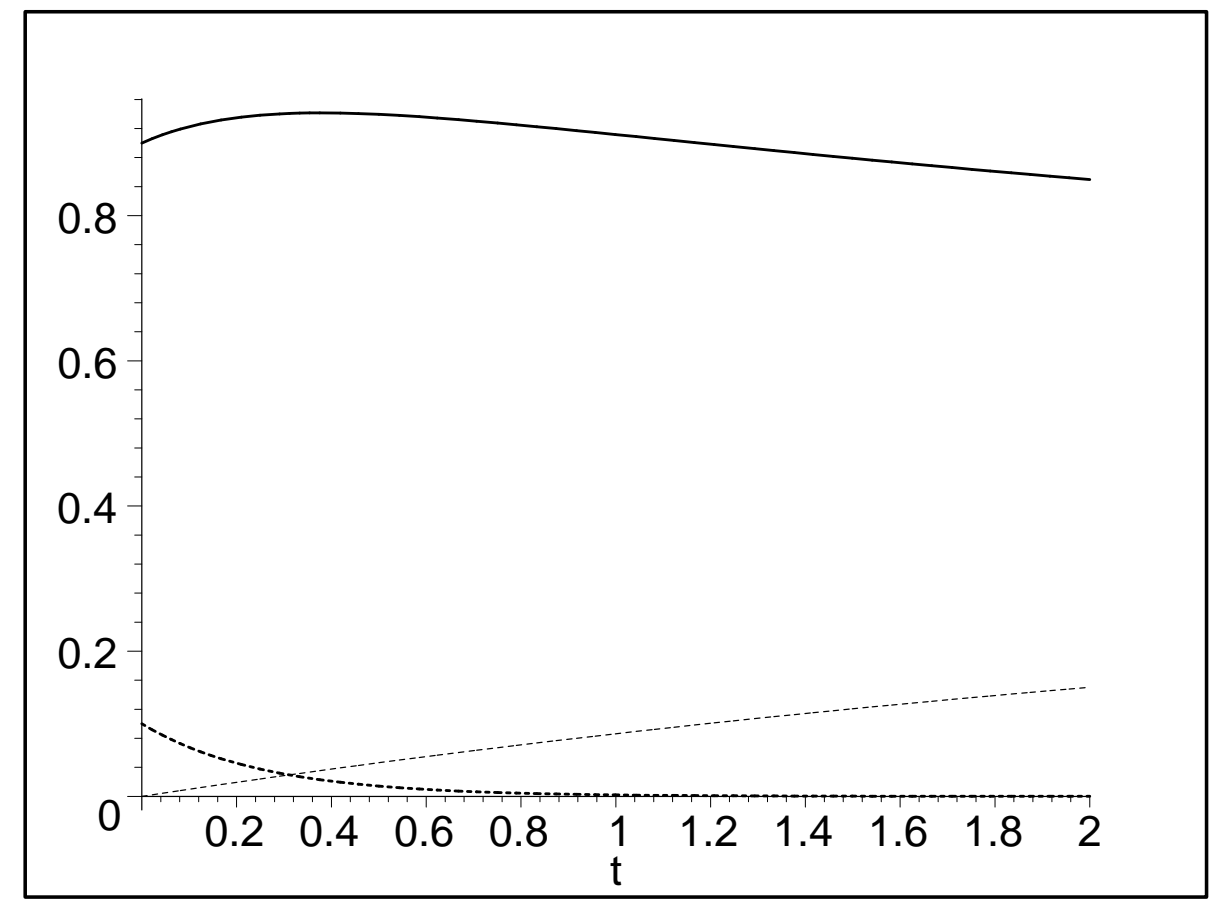

Fig. 1-A1: $\beta=\tilde{\beta}=0.3, \psi=0.1, S(0)=0.9, V(0)=0, I(0)=0.1$

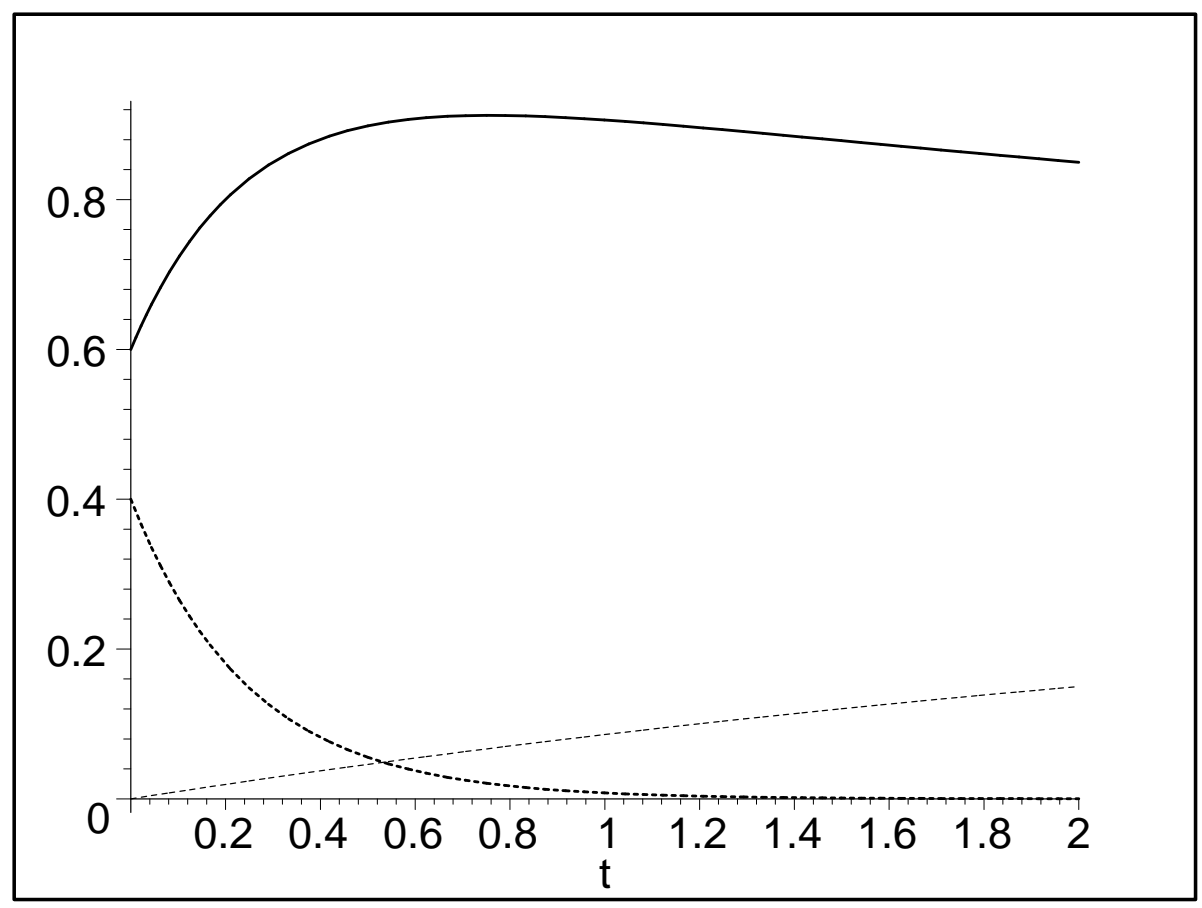

Fig. 1-B1: $\beta=\tilde{\beta}=0.3, \psi=0.1, S(0)=0.6, V(0)=0, I(0)=0.4$ 


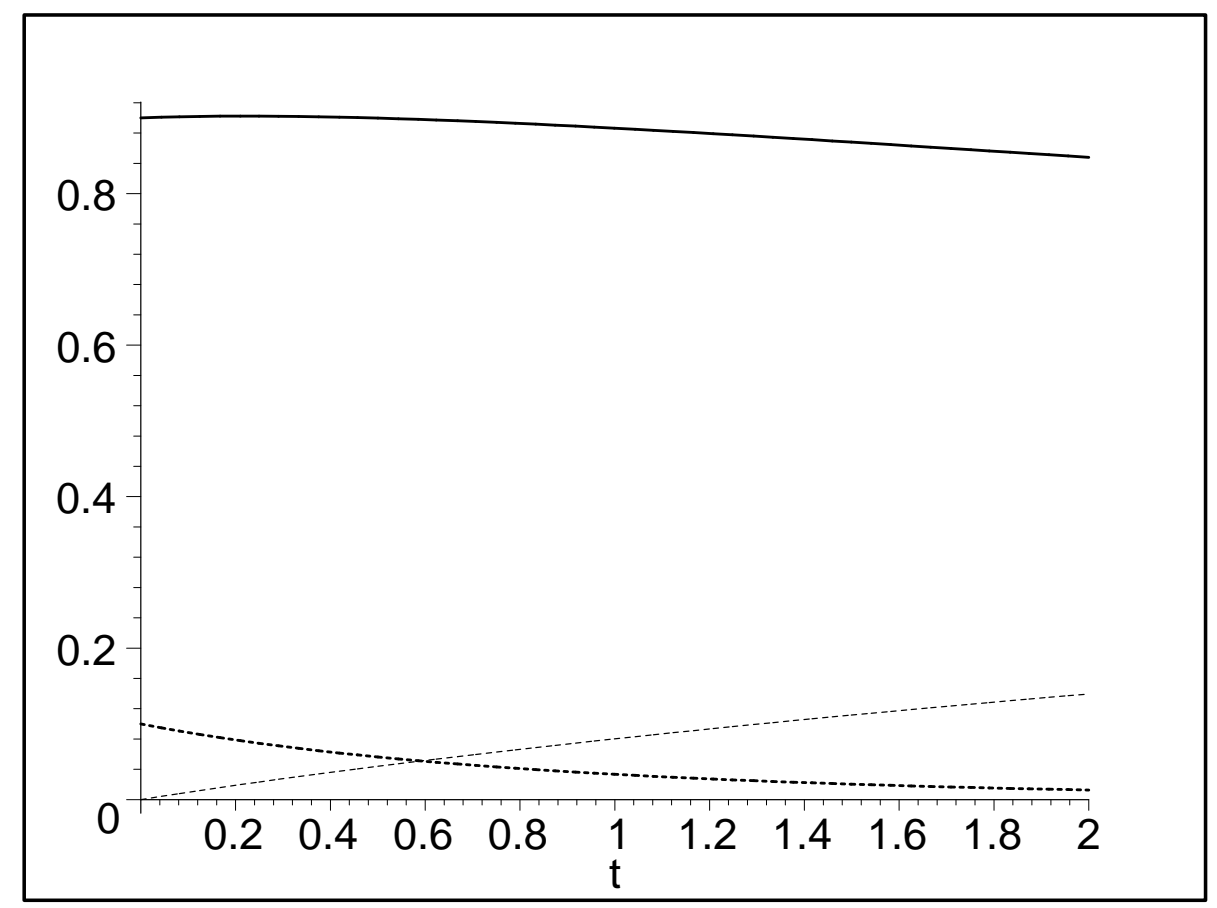

Fig. 1 -A2: $\beta=\tilde{\beta}=3.3, \psi=0.1, S(0)=0.9, V(0)=0, I(0)=0.1$ 


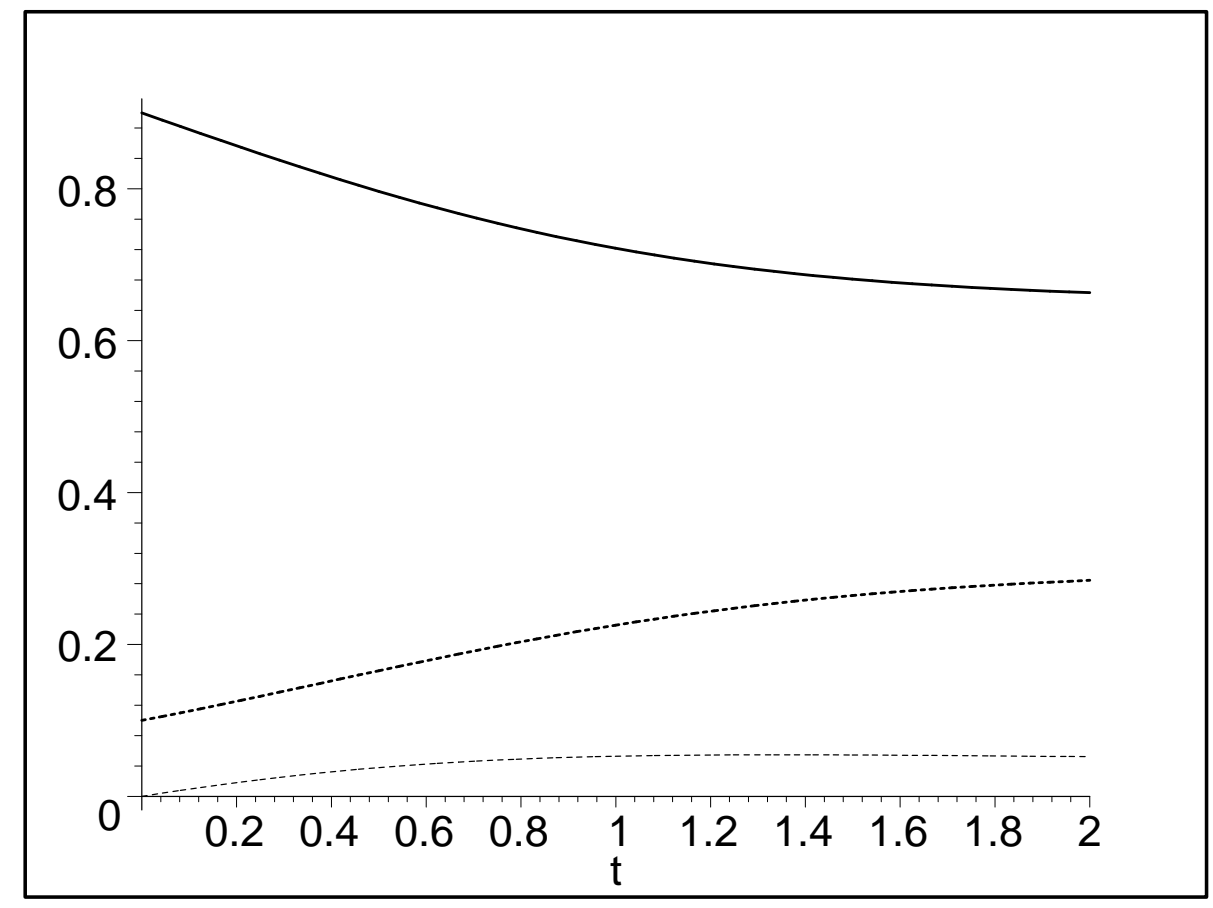

Fig. 1-A3: $\beta=\tilde{\beta}=6, \psi=0.1, S(0)=0.9, V(0)=0, I(0)=0.1$

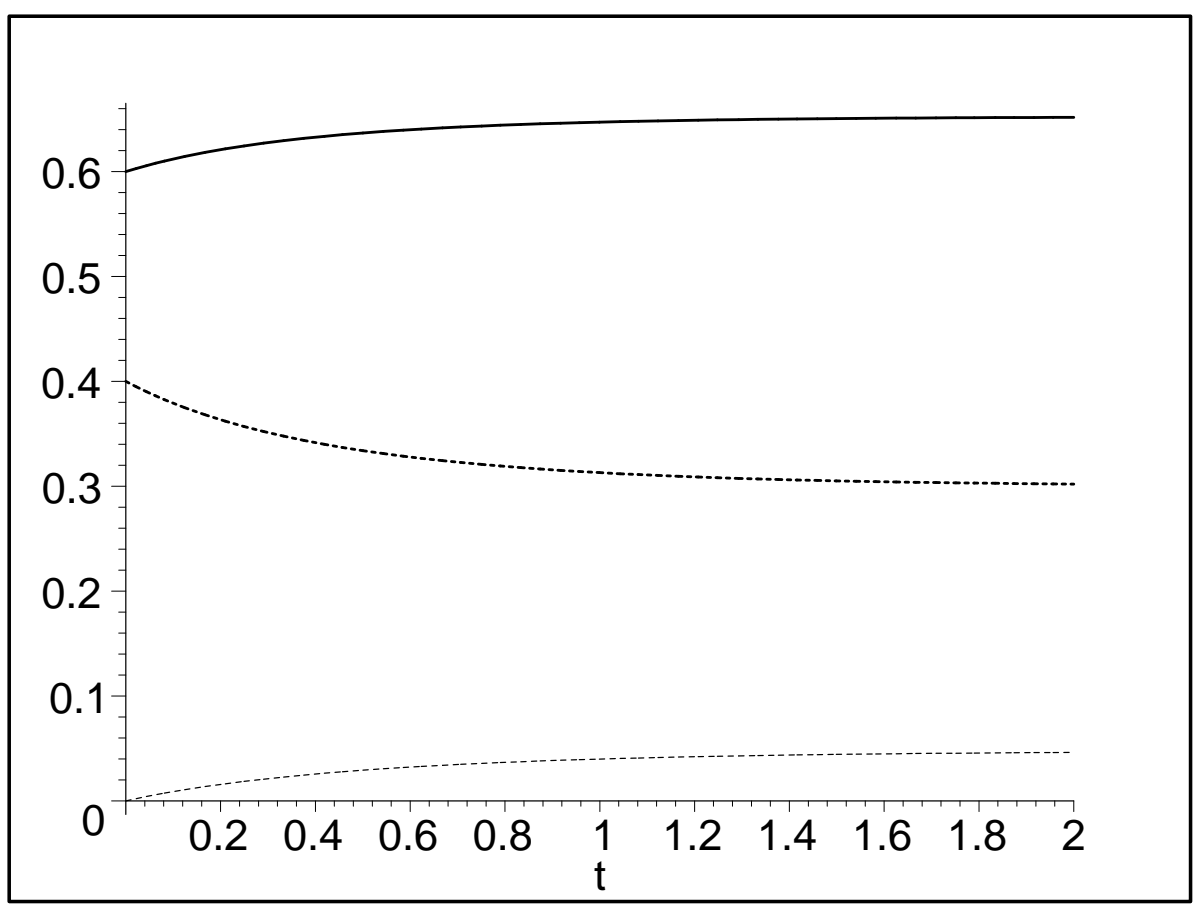

Fig. 1-B3: $\beta=\tilde{\beta}=6, \psi=0.1, S(0)=0.6, V(0)=0, I(0)=0.4$ 


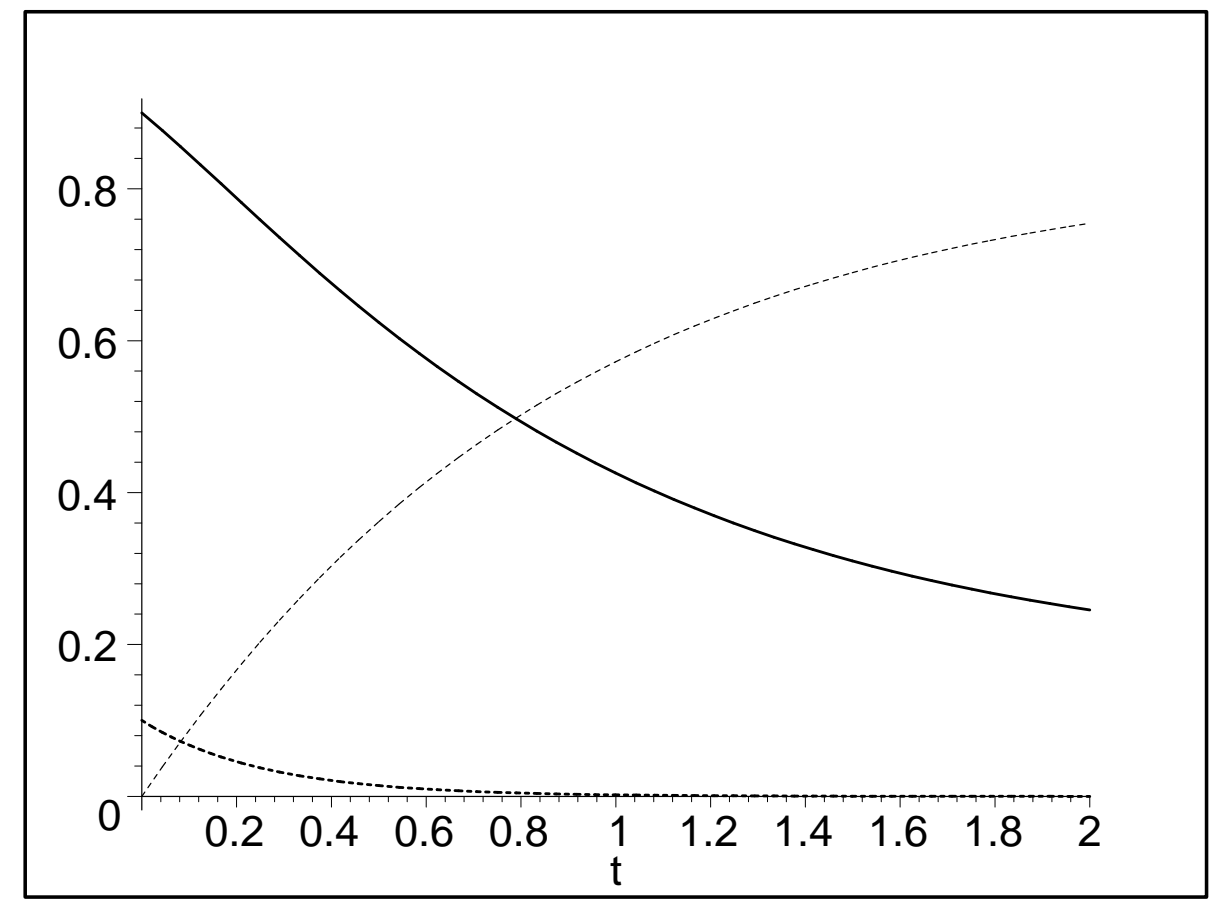

Fig. 1-A4: $\beta=\tilde{\beta}=0.3, \psi=1, S(0)=0.9, V(0)=0, I(0)=0.1$

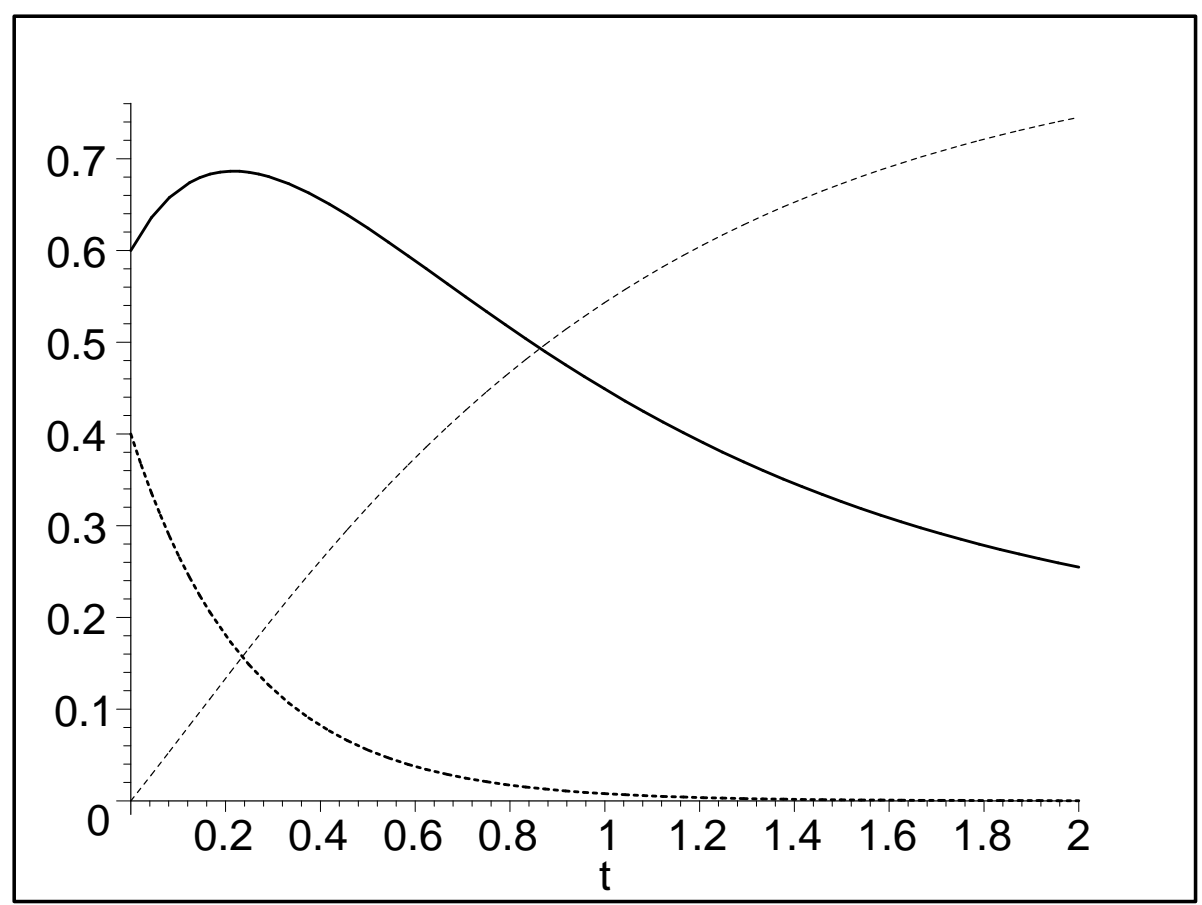

Fig. 1-B4: $\beta=\tilde{\beta}=0.3, \psi=1, S(0)=0.6, V(0)=0, I(0)=0.4$ 


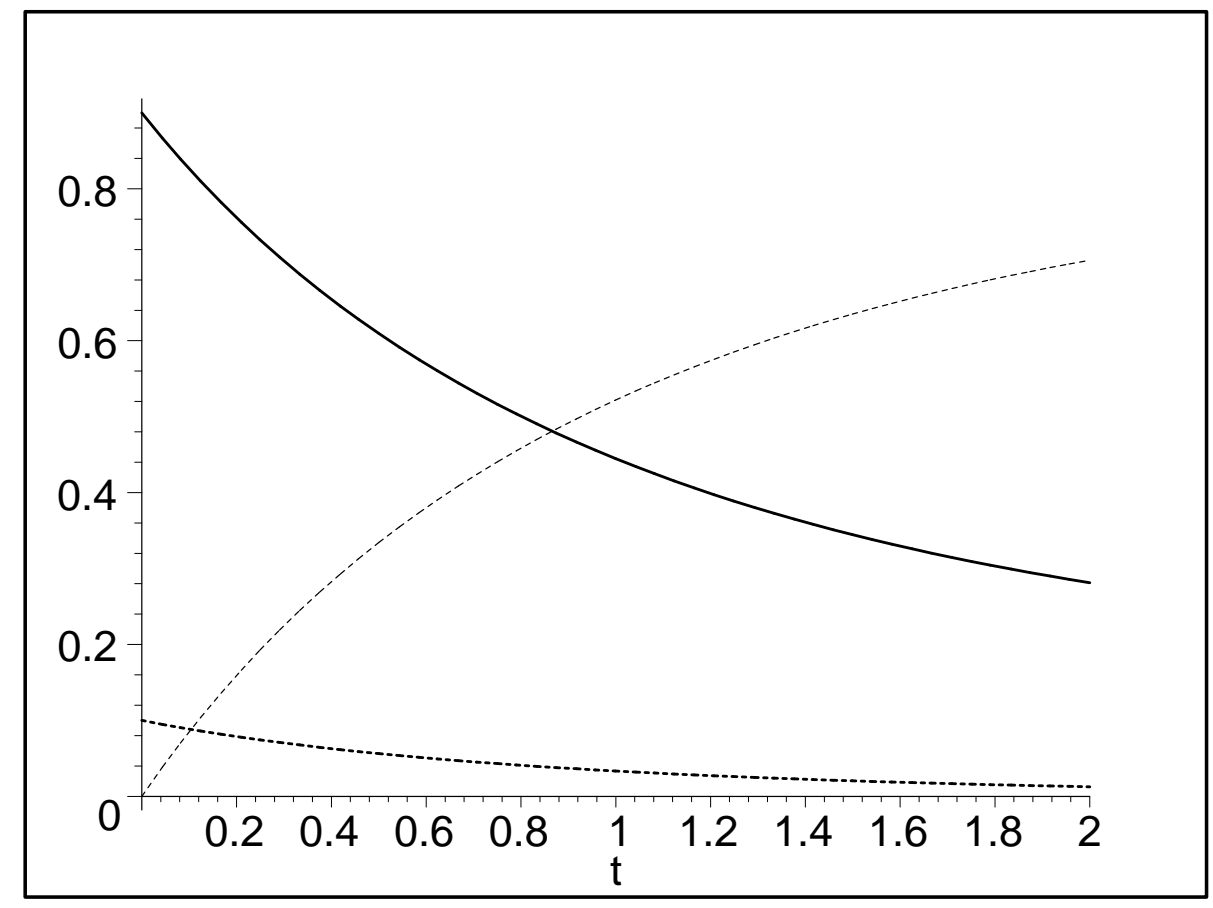

Fig. 1-A5: $\beta=\tilde{\beta}=3.3, \psi=1, S(0)=0.9, V(0)=0, I(0)=0.1$

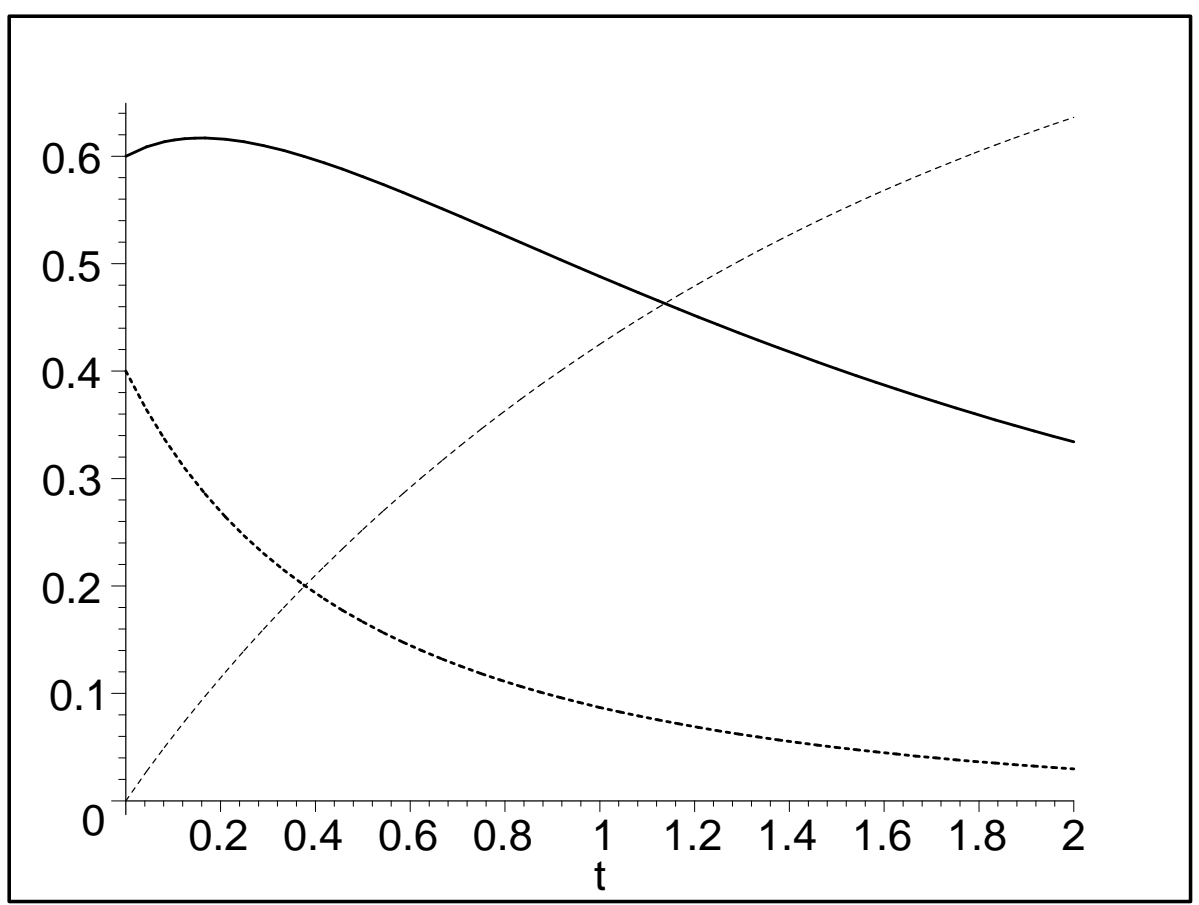

Fig. 1-B5: $\beta=\tilde{\beta}=3.3, \psi=1, S(0)=0.6, V(0)=0, I(0)=0.4$ 


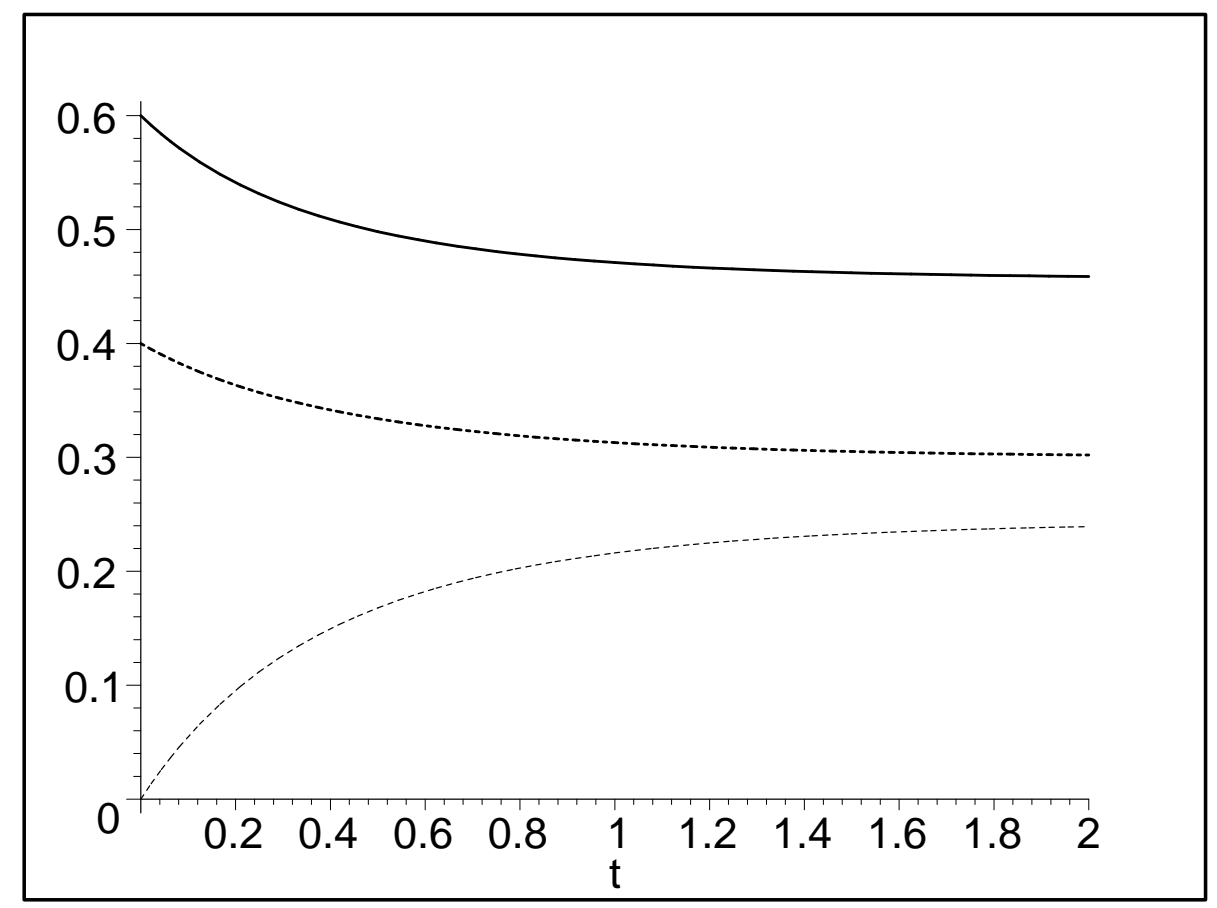

Fig. 1-B6: $\beta=\tilde{\beta}=6, \psi=1, S(0)=0.6, V(0)=0, I(0)=0.4$ 


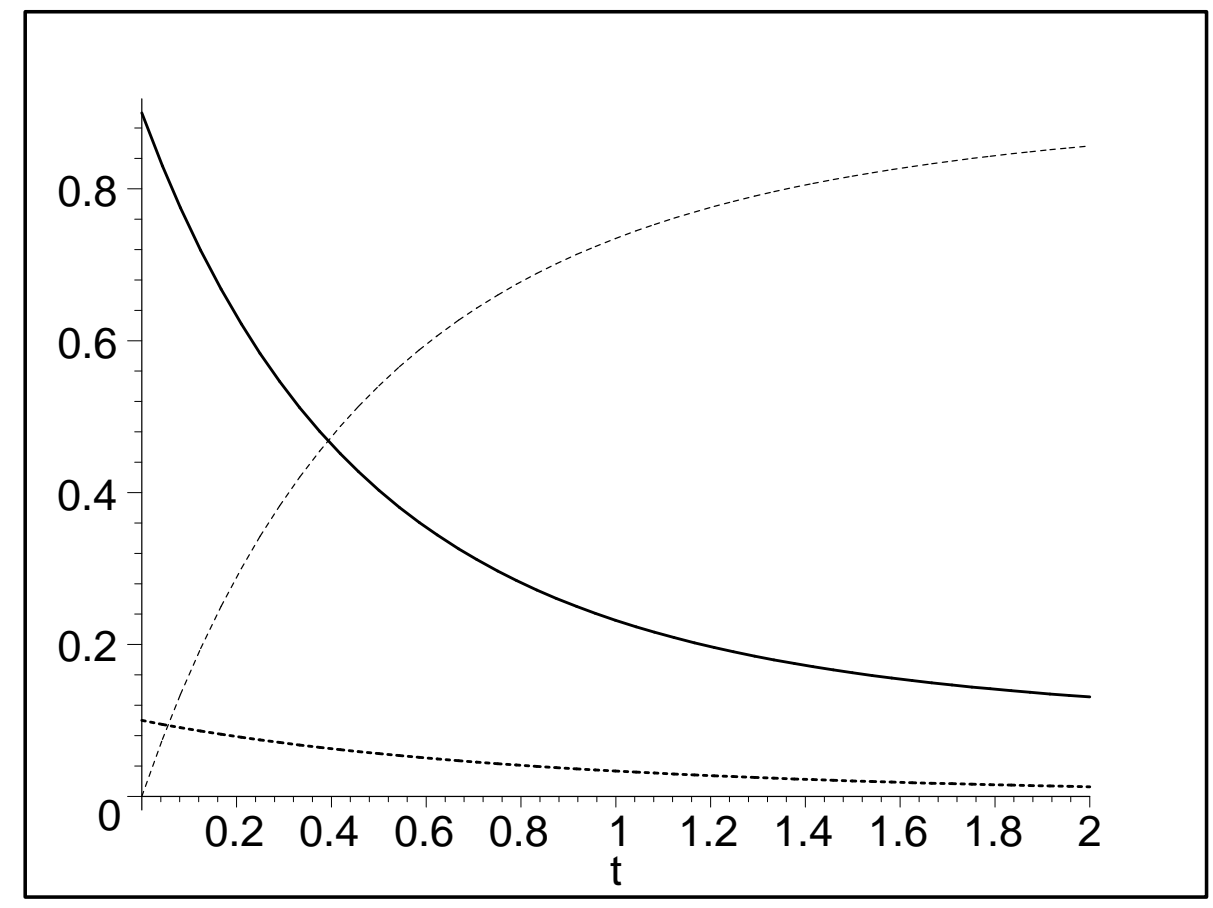

Fig. 1-A8: $\beta=\tilde{\beta}=3.3, \psi=2, S(0)=0.9, V(0)=0, I(0)=0.1$

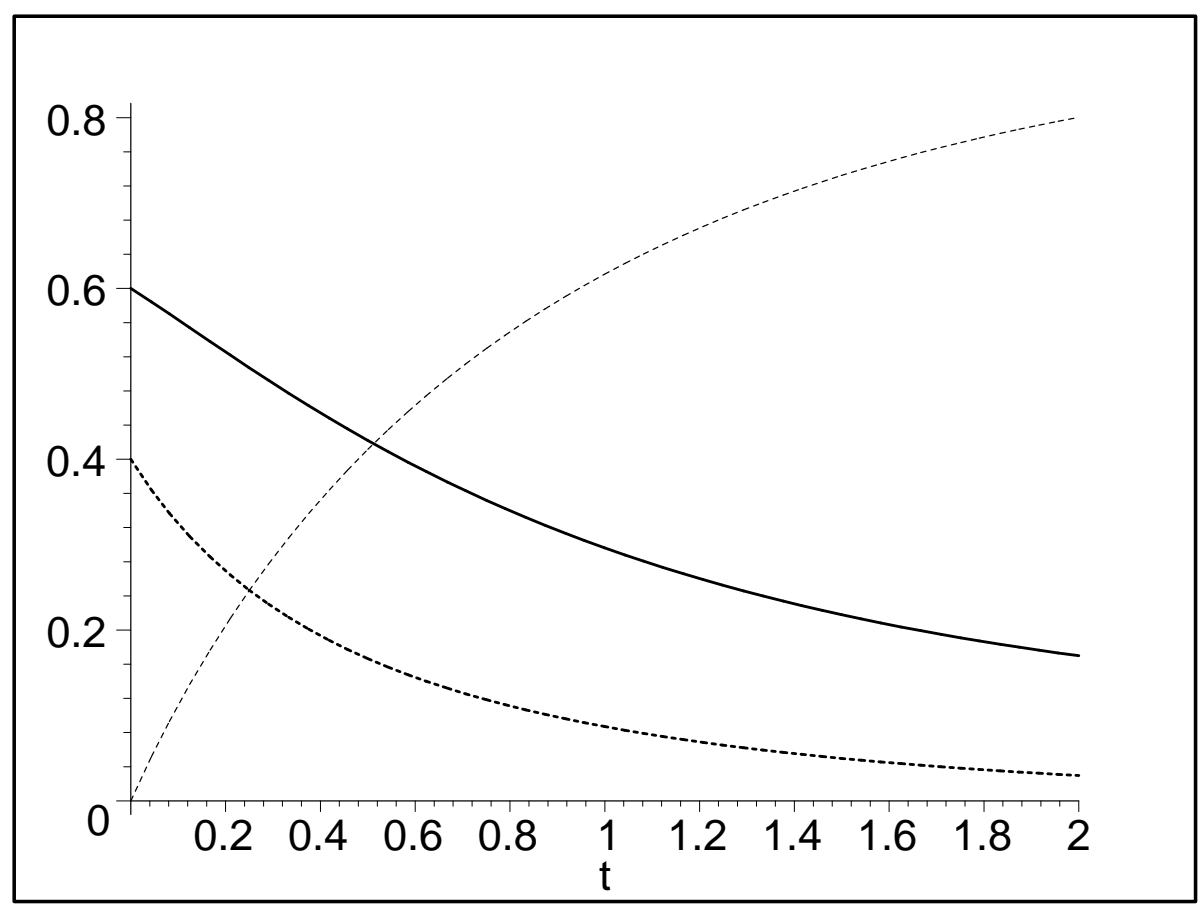

Fig. 1-B8: $\beta=\tilde{\beta}=3.3, \psi=2, S(0)=0.6, V(0)=0, I(0)=0.4$ 


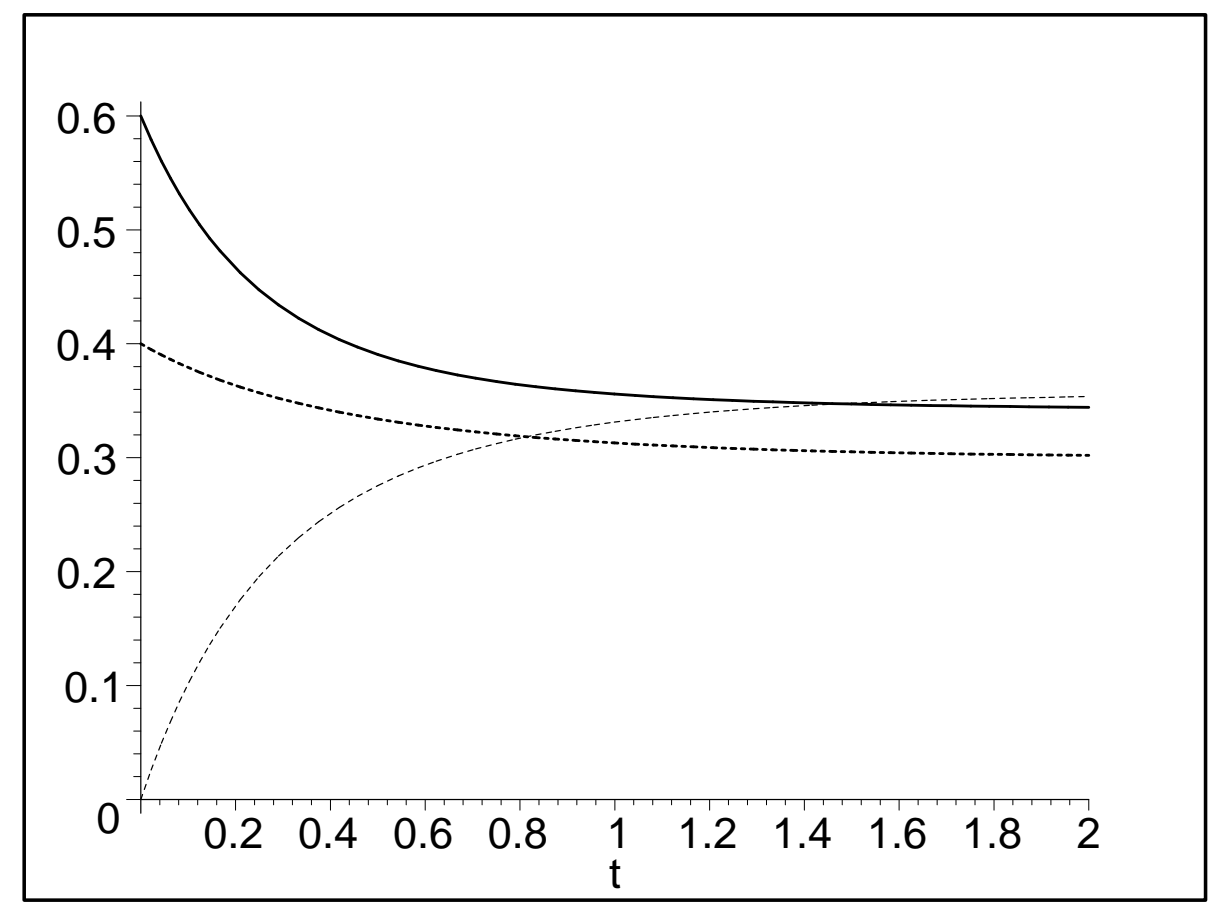

Fig. 1-B9: $\beta=\tilde{\beta}=6, \psi=2, S(0)=0.6, V(0)=0, I(0)=0.4$

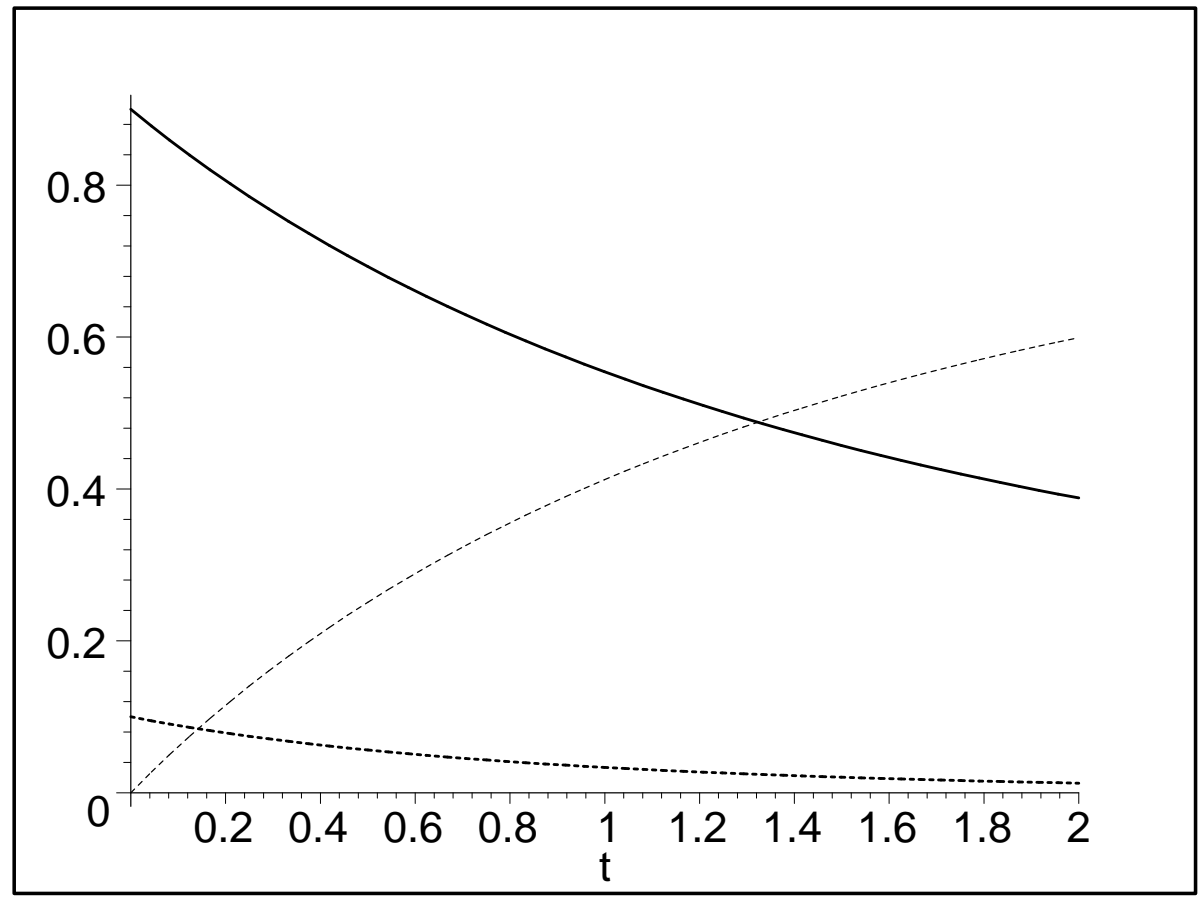

Fig. 1s1-A1: $\beta=\tilde{\beta}=3.3, \psi=0.7, S(0)=0.9, V(0)=0, I(0)=0.1$ 


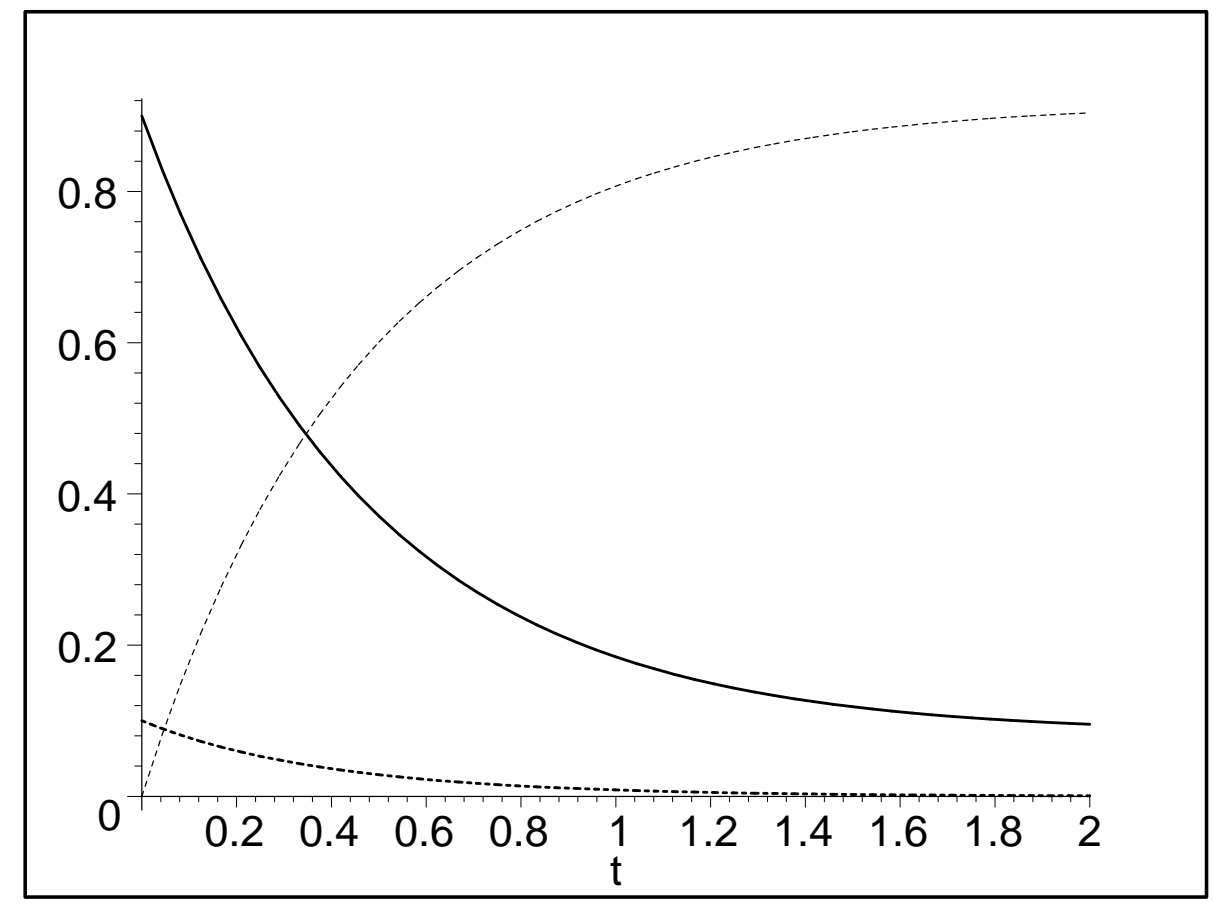

Fig. 1s1-A2: $\beta=\tilde{\beta}=1.8, \psi=2.2, S(0)=0.9, V(0)=0, I(0)=0.1$

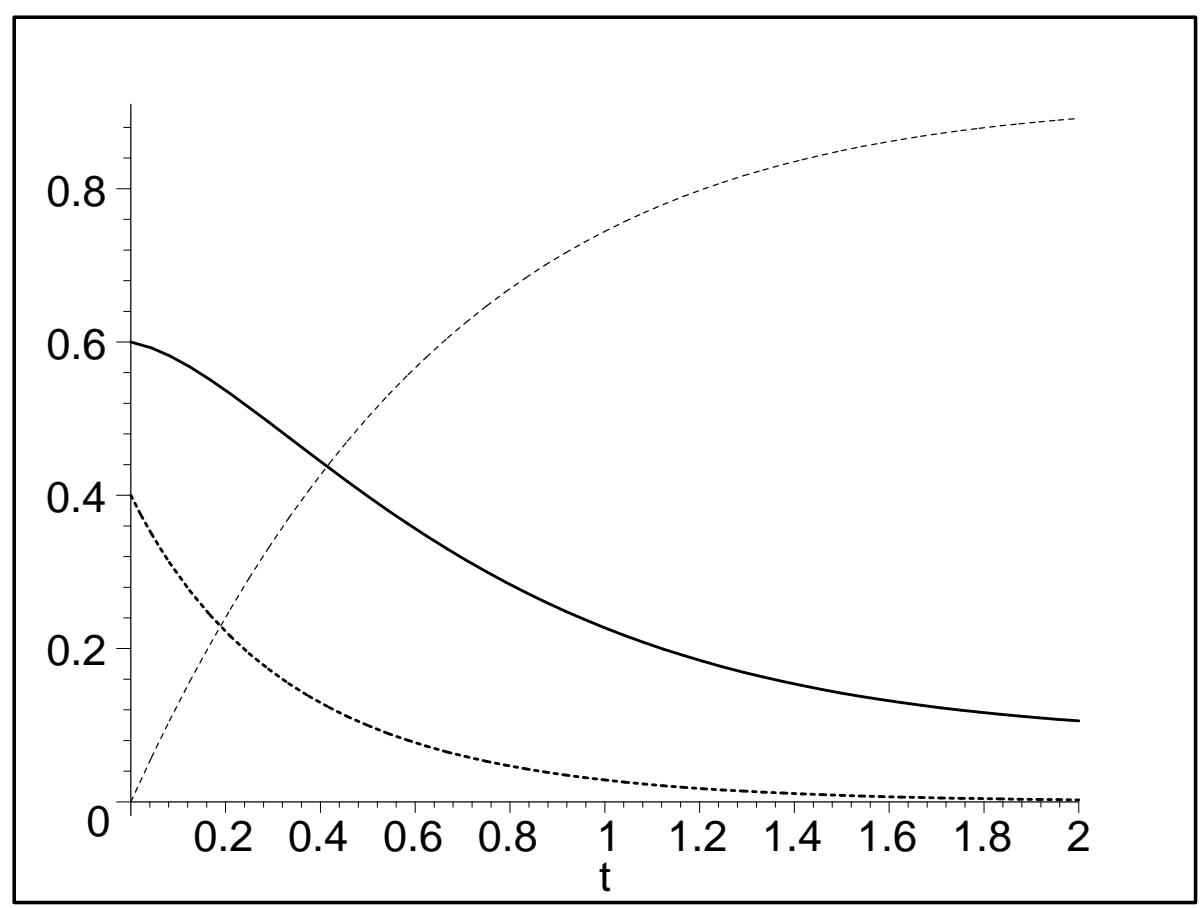

Fig. 1s1-B2: $\beta=\tilde{\beta}=1.8, \psi=2.2, S(0)=0.6, V(0)=0, I(0)=0.4$ 


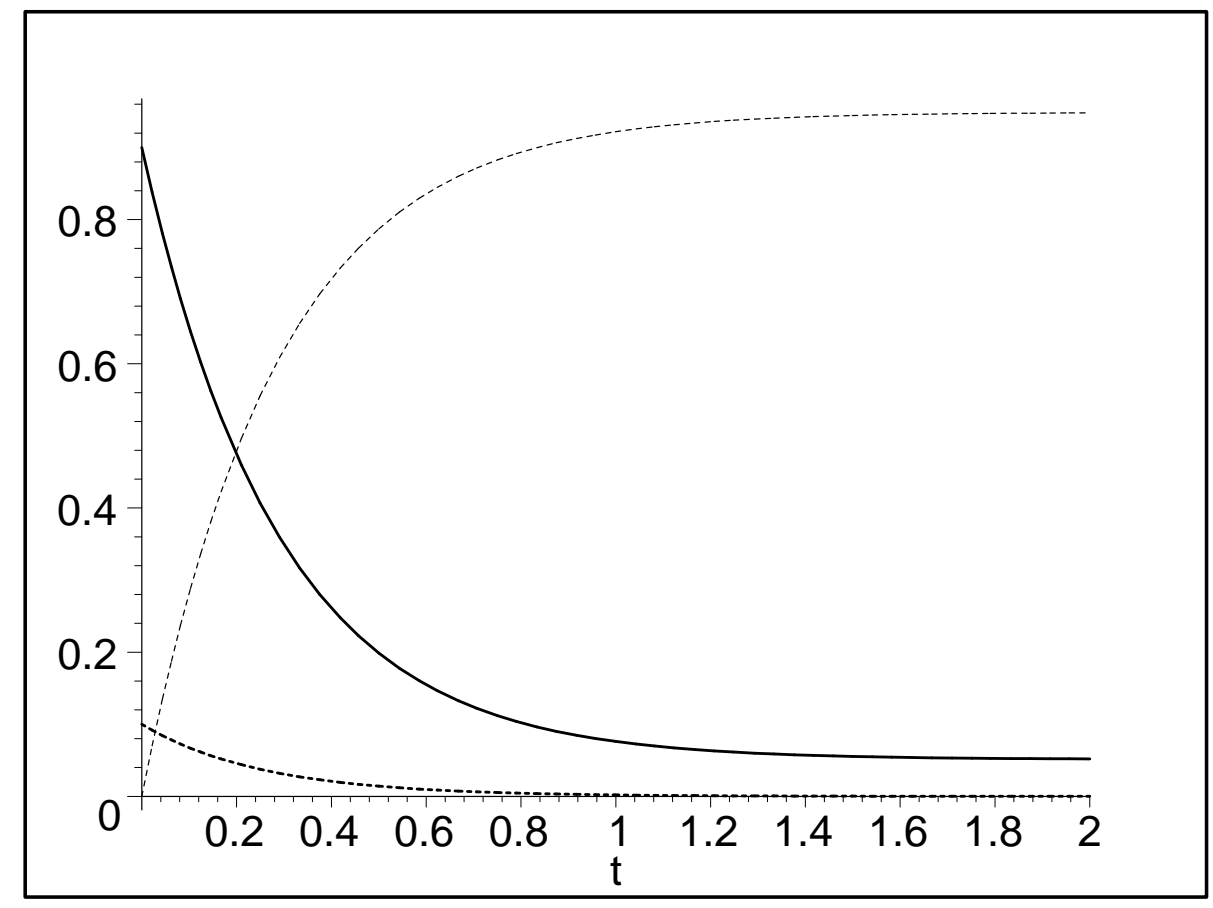

Fig. 1s1-A3: $\beta=\tilde{\beta}=0.3, \psi=3.7, S(0)=0.9, V(0)=0, I(0)=0.1$

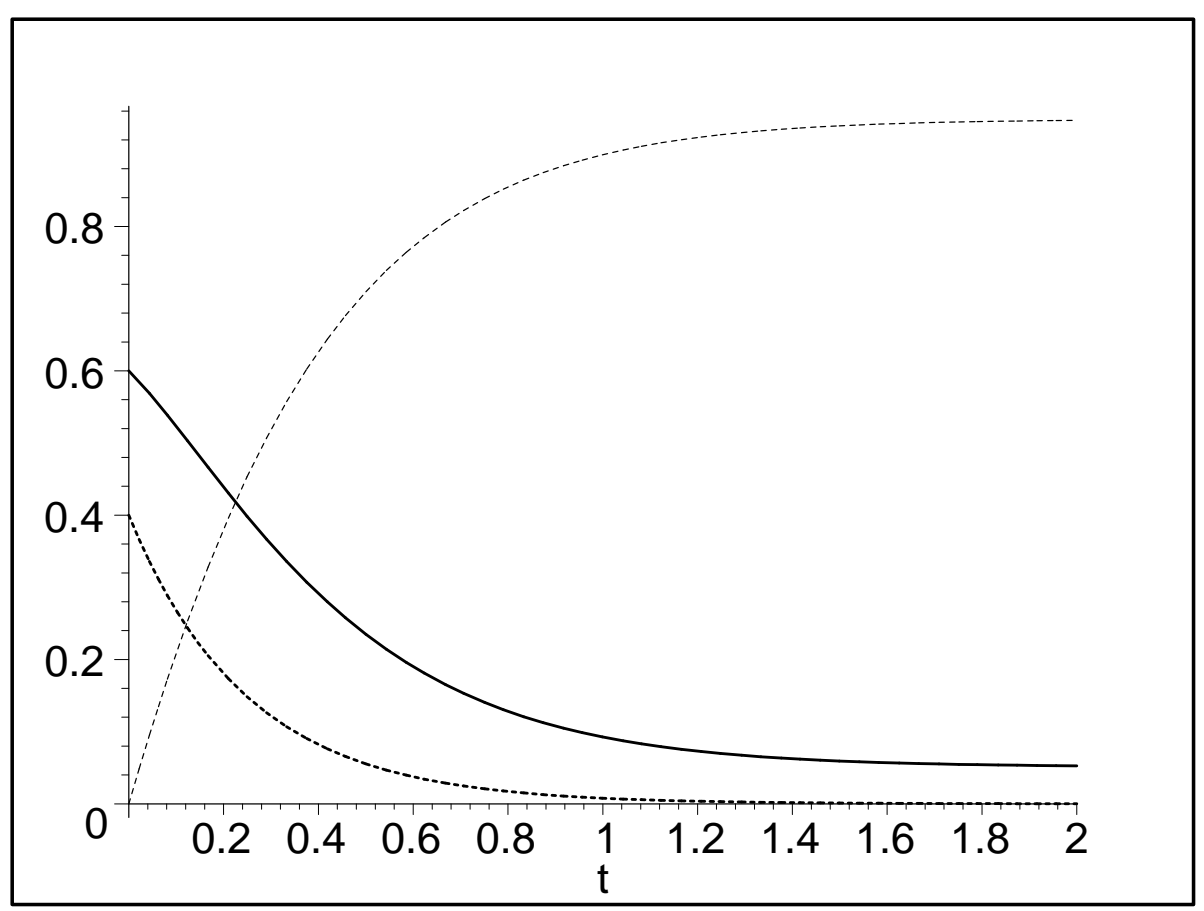

Fig. 1s1-B3: $\beta=\tilde{\beta}=0.3, \psi=3.7, S(0)=0.6, V(0)=0, I(0)=0.4$ 


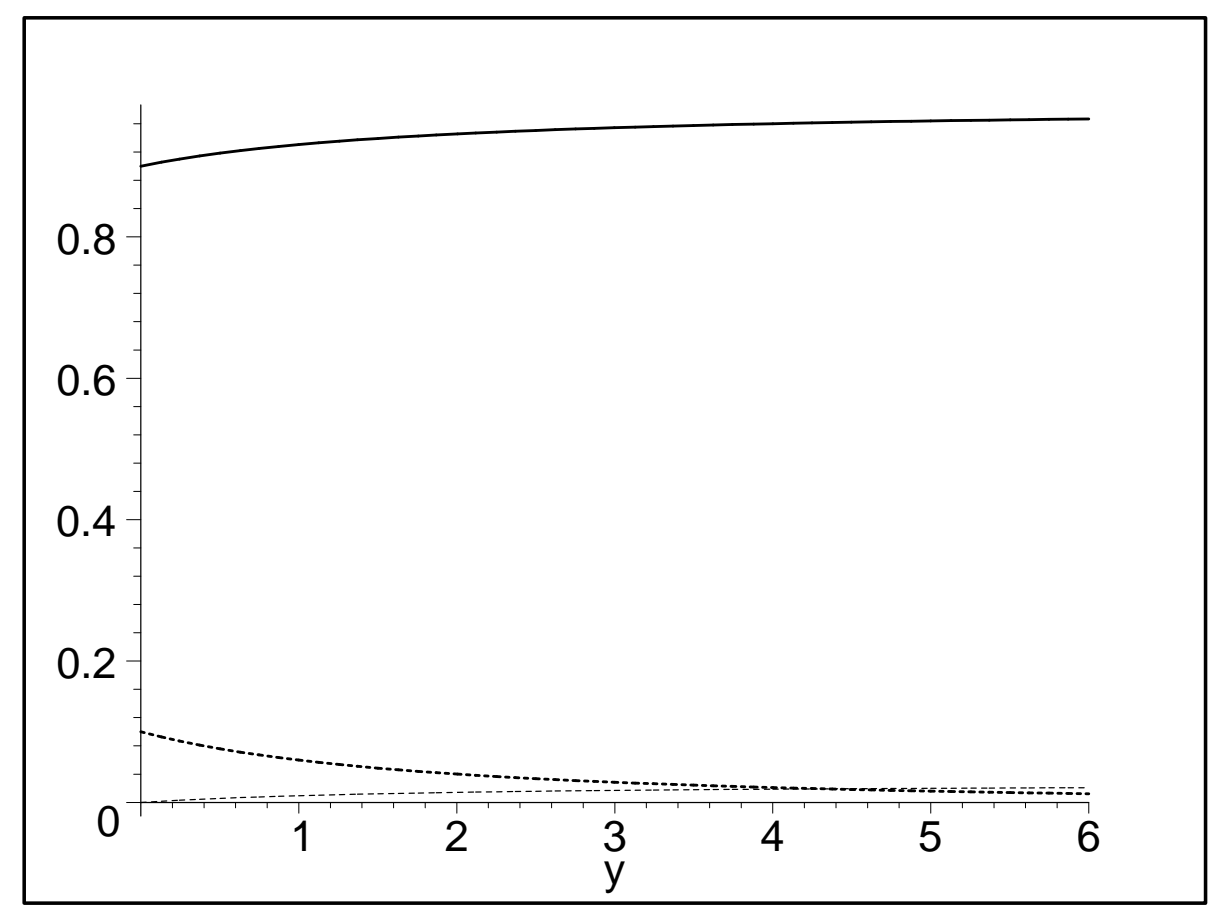

Fig. 1s2-A: $\beta=\tilde{\beta}=3.9951, \psi=0.004878, S(0)=0.9, V(0)=0, I(0)=0.1$

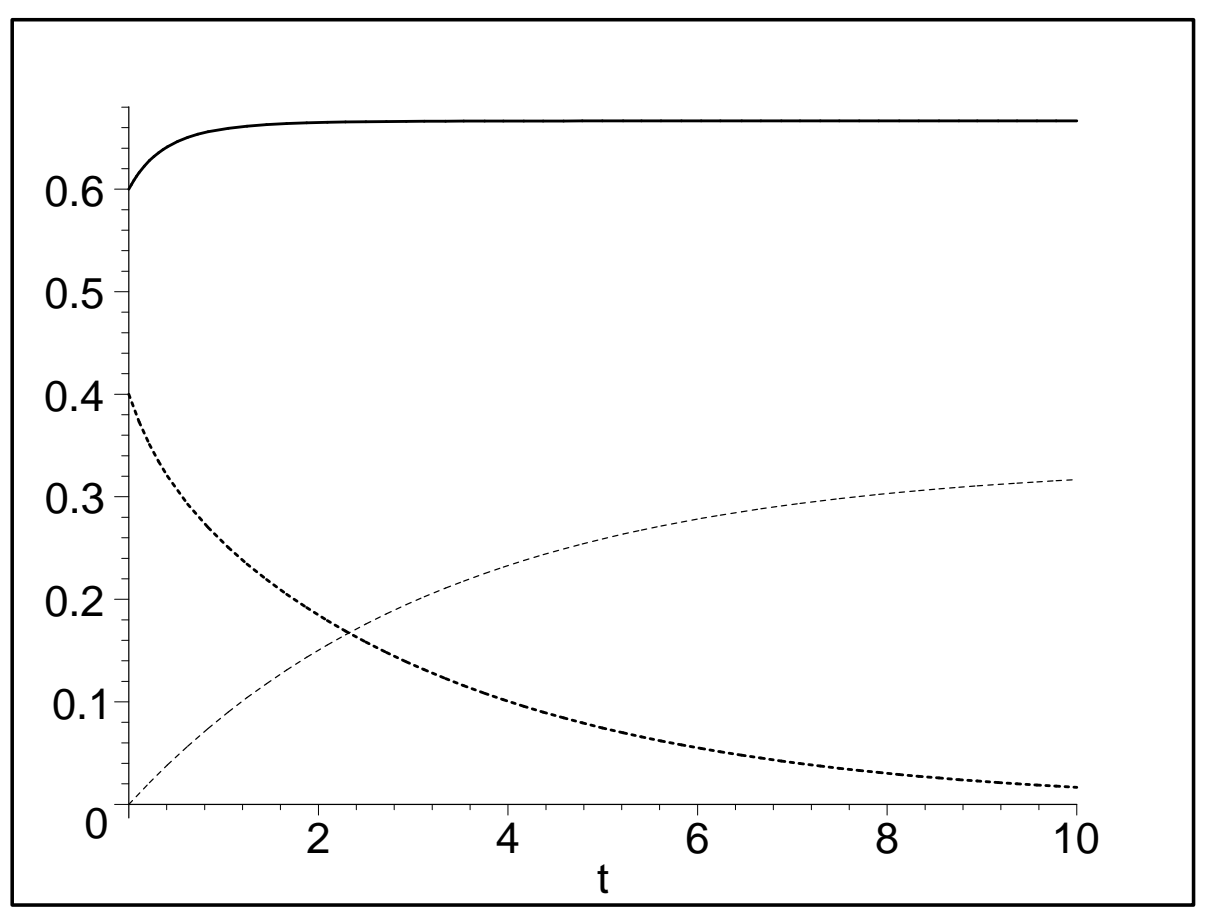

Fig. 5 -B: $\tilde{\beta}=0, \psi=0.1, \beta=5.85, S(0)=0.6, V(0)=0, I(0)=0.4$ 